\title{
BOUSSINESQ-PEREGRINE WATER WAVE MODELS AND THEIR NUMERICAL APPROXIMATION
}

\author{
THEODOROS KATSAOUNIS, DIMITRIOS MITSOTAKIS, AND GEORGE SADAKA
}

\begin{abstract}
In this paper we consider the numerical solution of Boussinesq-Peregrine type systems by the application of the Galerkin finite element method. The structure of the Boussinesq systems is explained and certain alternative nonlinear and dispersive terms are compared. A detailed study of the convergence properties of the standard Galerkin method, using various finite element spaces on unstructured triangular grids, is presented. Along with the study of the Peregrine system, a new Boussinesq system of BBM-BBM type is derived. The new system has the same structure in its momentum equation but differs slightly in the mass conservation equation compared to the Peregrine system. Further, the finite element method applied to the new system has better convergence properties, when used for its numerical approximation. Due to the lack of analytical formulas for solitary wave solutions for the systems under consideration, a Galerkin finite element method combined with the Petviashvili iteration is proposed for the numerical generation of accurate approximations of line solitary waves. Various numerical experiments related to the propagation of solitary and periodic waves over variable bottom topography and their interaction with the boundaries of the domains are presented. We conclude that both systems have similar accuracy when approximate long waves of small amplitude while the Galerkin finite element method is more efficient when applied to BBM-BBM type systems.
\end{abstract}

\section{INTRODUCTION}

Two identifying physical properties of water waves are the nonlinearity and dispersion. Dispersion refers to the property of waves of different wavelength to travel with different phase speed, while the nonlinearity is related to the way waves interact with each other and also with the fact that waves of larger amplitude propagate faster than those of smaller amplitude. The balanced combination of these two properties is the basic ingredient for the existence of solitary waves. In fluid mechanics the propagation of water waves is described by the Euler equations of ideal fluids (inviscid and irrotational) [47]. Due to the complexity of the Euler equations we usually rely on numerical simulations of simplified mathematical models consisting of nonlinear and dispersive partial differential equations when studying the properties of water waves. Depending on the nature of the waves we study, there are different mathematical models to describe their propagation. For example there are different mathematical models for long waves, short waves, small amplitude or large amplitude waves, waves in small, intermediate or large water depth.

In this paper we focus on some mathematical models derived especially to describe the propagation of small amplitude, long waves compared to the depth of the water. If $A$ denotes a typical wave amplitude in a general state, $D_{0}$ a typical water depth and $\lambda$ a typical wavelength, then waves that satisfy $\varepsilon:=A / D_{0} \ll 1$ and $\sigma:=D_{0} / \lambda \ll 1$ are characterized as long waves of small amplitude in the shallow water regime. Moreover, usually it is required that the Stokes number is $S:=\varepsilon / \sigma^{2}=O(1)$. Examples of such waves are the important cases of solitary waves and tsumamis in deep ocean waters. The mathematical modelling of water waves of small amplitude in a realistic environment with general bathymetry was initiated by Peregrine [39], by deriving asymptotically 
the so called Peregrine system

$$
\begin{aligned}
& \eta_{t}+\nabla \cdot[(D+\eta) \mathbf{u}]=0, \\
& \mathbf{u}_{t}-\frac{1}{2} D \nabla\left(\nabla \cdot\left(D \mathbf{u}_{t}\right)\right)+\frac{1}{6} D^{2} \nabla\left(\nabla \cdot \mathbf{u}_{t}\right)+g \nabla \eta+(\mathbf{u} \cdot \nabla) \mathbf{u}=0, \quad \text { Classical Peregine, }
\end{aligned}
$$

where $\eta(\mathbf{x}, t)=\eta(x, y, t)$ denotes the free-surface elevation above an undisturbed level and $\mathbf{u}(\mathbf{x}, t)=$ $(u(x, y, t), v(x, y, t))$ is the depth averaged horizontal velocity vector field of the water over a variable bottom with depth $D(\mathbf{x})=D(x, y)>0$. A sketch of the domain is depicted in Figure 1 . It is

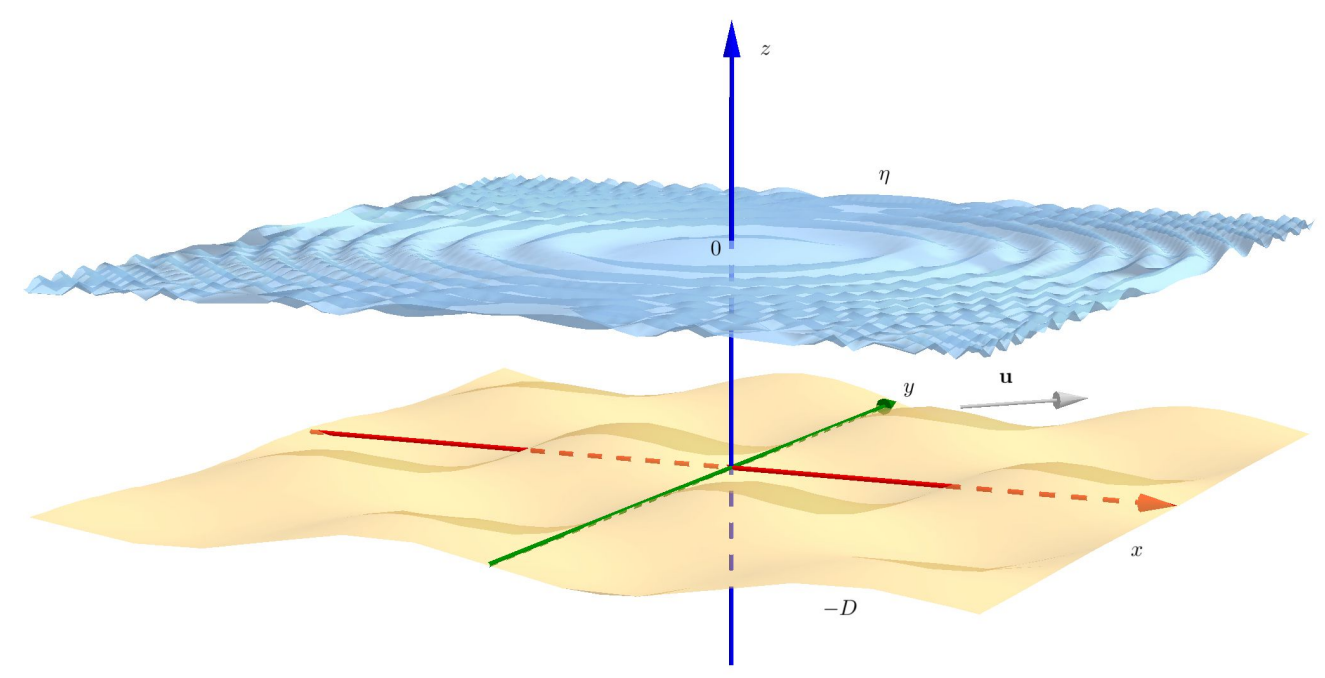

Figure 1. Sketch of the physical domain

noted that $\mathbf{u}$ can be thought of as the horizontal velocity of the fluid at a certain height above the bottom.

Peregrine's system is known to possess solitary wave solutions similar to the Euler equations [23] and served as a base for the study of water waves, [20]. As a mathematical model, it can be derived using asymptotic techniques and thus has a different linear dispersion relation from that of the Euler equations. Although it is expected to model accurately long waves of small amplitude, there are special cases where the linear dispersion relation is important for the accurate approximation of water waves, [21]. Based on the idea of evaluating the horizontal velocity of the fluid at any depth [15], an alternative Boussinesq system was derived in the same regime with improved dispersion relation characteristics [36]

$$
\begin{aligned}
& \eta_{t}+\nabla \cdot((D+\eta) \mathbf{u})+\nabla \cdot\left[\tilde{a} D^{2} \nabla(\nabla \cdot(D \mathbf{u}))+\tilde{b} D^{3} \nabla(\nabla \cdot \mathbf{u})\right]=0, \\
& \mathbf{u}_{t}+g \nabla \eta+(\mathbf{u} \cdot \nabla) \mathbf{u}+\tilde{c} D \nabla\left(\nabla \cdot\left(D \mathbf{u}_{t}\right)\right)+\tilde{d} D^{2} \nabla\left(\nabla \cdot \mathbf{u}_{t}\right)=0,
\end{aligned}
$$

where now $\mathbf{u}$ denotes the horizontal velocity vector field evaluated at height $z=-D+\theta(\eta+D)$ for $\theta \in[0,1]$, and $\tilde{a}=\theta-1 / 2, \tilde{b}=1 / 2\left[(\theta-1)^{2}-1 / 3\right]$ ), $\tilde{c}=\theta-1$ and $\tilde{d}=1 / 2(\theta-1)^{2}$ (see also $[34,36])$. 
The finite element method for the numerical solution of the Peregrine system (1) has been introduced in several occasions to study practical problems in coastal engineering $[10,5,32,43,48]$. Due to the simplicity of the mathematical formulation of Peregrine's system, the formulation of the standard Galerkin finite element method in a bounded domain and with slip-wall boundary conditions such as $\mathbf{u} \cdot \mathbf{n}=0$, with $\mathbf{n}$ being the unit outward normal vector on the boundary of the computational domain, is straightforward. On the other hand its convergence properties for the specific model still remain unknown.

The standard Galerkin finite element method for the Peregrine system has been studied analytically only in one-dimension and has been proven that its convergence is suboptimal when the same finite element space is used for both dependent variables and it is optimal when different finite element spaces for the approximation of the free-surface elevation and the horizontal velocity are considered $[7,8,6]$. The two-dimensional case is different though, and the main reason is that the regularization operator in the momentum conservation of Peregrine's system is the $I-\nabla(\nabla \cdot)$. This operator in one-dimension is identical to the Laplace operator, but in two dimensions is not fully-elliptic as some of the second derivatives are missing. For example, if the solution $\mathbf{u}$ is restricted in the curl vector field space, then this operator is just the identity. For this reason we will call this operator incomplete-elliptic operator. Its inversion then can create implications as far as it concerns the regularity of the solution as well as the convergence properties of the numerical method. It is noted that Nwogu's system (with optimal in some sense linear dispersion properties [36]) contains the same incomplete-elliptic operator in the momentum conservation equation and thus similar behaviour in the convergence properties of the variable $\mathbf{u}$ is expected.

Another reason for observing suboptimal convergence rates, in the case of Peregrine's system is the form of the mass conservation equation (1), which resembles a first order hyperbolic equation. While in Nwogu's system the mass conservation equation is not a hyperbolic equation, the presence of the third-order spatial derivative is expected to cause different problems in the finite element semidiscretization and especially complications on the implementation of additional boundary conditions [46]. For the numerical implementation of a finite element method for Nwogu's system we refer to [45]. These peculiarities have been realised in [34] where Boussinesq systems were modified by using the irrotationality condition that governs ideal fluids with potential flow. A particular regularized system of BBM-BBM type was derived in [34] in the form

$$
\begin{aligned}
& \eta_{t}+\nabla \cdot[(D+\eta) \mathbf{u}]-\frac{1}{6} \nabla \cdot\left(D^{2} \nabla \eta_{t}\right)=0, \\
& \mathbf{u}_{t}+g \nabla \eta+(\mathbf{u} \cdot \nabla) \mathbf{u}-\frac{1}{6} D^{2} \Delta \mathbf{u}_{t}=0 .
\end{aligned}
$$

This system is analogous to the BBM-BBM system derived in [14] in the flat bottom case. The BBM(Benjamin-Bona-Mahony), [12] terminology refers to the presence in the model of a third order term with two spatial and one time derivative, like the last two terms in (3).

Although the finite element method for the specific system appears to converge with optimal rate, the disadvantage of the specific regularized system is that the inversion of the elliptic operator requires non-slip wall boundary conditions of the form $\mathbf{u}=0$ or the computation of the tangential component of the velocity vector field on the boundary of the domain $[24,25]$. Non-slip wall boundary conditions can be restrictive while the computation of the tangential component of the velocity on the boundary of the domain can be rather complicated. On the other hand, such BBMBBM type system was shown to have very good performance in studies of water waves over variable bottom $[42,28,34]$ and for this reason certain improvements can be made so as to make slip-wall boundary conditions easily applicable.

In this paper we compare computationally various Peregrine type models, reveal their qualitative and quantitive characteristics and evaluate their ability to model the propagation of water waves 
of small amplitude and long wavelength. In particular, we study computationally the convergence properties of the standard Galerkin finite element method for the Peregrine system (1) and we show that the effects of the hyperbolic form of the mass conservation results in suboptimal convergence rates for the surface elevation. Optimal convergence rates can be obtained only by using different finite element spaces between the free-surface elevation and the velocity field. We also show that optimal convergence rates for the velocity variable can be achieved by replacing the incompleteelliptic regularization operator in the momentum conservation equation by the Laplace operator as in system (3). This substitution can be explained using asymptotic arguments. Of course such modification will inflict again restrictive zero Dirichlet boundary condition on the velocity vector field. We also show that the nonlinearity has no implication on the convergence properties of the numerical method. In particular, modifying the nonlinearity that appears in the momentum conservation equation to one given in conservative form using the same asymptotic arguments we observed the same convergence properties.

Finally, we derive a new form of the BBM-BBM system, (3) which is appropriate for slip-wall boundary conditions. The new system appears to have optimal convergence rates for the freesurface elevation variable. The momentum conservation equation retains the same mathematical formulation as in the Peregrine system and thus slip-wall boundary conditions can be used in a straightforward manner. The new system appears to be highly accurate in the appropriate shallow water regime and due to the better convergence properties it can be used as an alternative system for the computations of long water waves of small amplitude. The numerical models are validated against standard benchmarks where the numerical solutions are compared with laboratory data. For the numerical generation of accurate approximation of solitary waves in the experiments we describe a Galerkin finite element method combined with the Petviashvili iteration [40] for the solution of the resulting nonlinear equations. To the best of our knowledge this is the first time that the Petviashvili method is combined successfully with the finite element method to produce approximate solitary waves, for a system of equations in two space dimensions.

The structure of the paper is as follows: In Section 2 after reviewing the Peregrine system and its properties, we derive the version of the Peregrine system with the standard elliptic regularization operator and the modified nonlinearity. In the same section we derive also the new BBM-BBM type system, which is appropriate for slip-wall boundary conditions. Section 3 is devoted to the study of the convergence properties of Finite Element Methods for the various Boussinesq systems. The numerical generation of solitary waves is presented in Section 4 and further testing of the accuracy of the numerical methods on the propagation of solitary waves is considered. The numerical generation of solitary waves is based on the Petiasvili method described in the Appendix A. Section 5 presents standard benchmark experiments for Boussinesq systems showing that all models with the same asymptotic behaviour result to very similar physically sound solutions. Conclusions and further developments are discussed in Section 6.

\section{Mathematical Models}

2.1. Notation. Let $\Omega \subset \mathbb{R}^{2}$ be a bounded set with smooth boundary $\Gamma=\partial \Omega$ and let $L^{2}(\Omega)$ denotes the standard Lebesque space of square integrable functions in $\Omega$. We shall use the standard notation for the Sobolev spaces defined on $\Omega$ and their corresponding norms:

$$
V:=H^{1}(\Omega)=\left\{\phi \in L^{2}(\Omega): \nabla \phi \in L^{2}(\Omega)\right\}, \quad\|\phi\|_{1}^{2}=\|\phi\|^{2}+\|\nabla \phi\|^{2},
$$

where $\|\cdot\|$ denotes the norm in $V,(\cdot, \cdot)$ is the corresponding inner product in $\Omega$ and $\langle\cdot, \cdot\rangle$ the inner product on $\Gamma$. The extension to vector fields is straightforward, i.e. if $\mathbf{u}=\left(u_{1}, u_{2}\right), \mathbf{v}=\left(v_{1}, v_{2}\right) \in$ $L^{2}(\Omega)^{2}$, then $(\mathbf{u}, \mathbf{v})=\sum_{i=1}^{2}\left(u_{i}, v_{i}\right)$ and $\|\mathbf{u}\|^{2}=\sum_{4}^{2}\left\|u_{i}\right\|^{2}$. Further we shall also work with the 
Sobolev spaces

$$
H(\operatorname{div}, \Omega)=\left\{\mathbf{v} \in L^{2}(\Omega)^{2}: \nabla \cdot \mathbf{v} \in L^{2}(\Omega)\right\}
$$

with norm

$$
\|\mathbf{v}\|_{\mathrm{H}(\text { div })}^{2}=\|\mathbf{v}\|^{2}+\|\nabla \cdot \mathbf{v}\|^{2} .
$$

The bottom topography is described by $D(\mathbf{x})$ and is independent of time. Further, we assume throughout this work that $D(\mathbf{x})$ is smooth and there are positive constants $D_{m}, D_{M}$ such that $0<D_{m} \leq D(\mathbf{x}) \leq D_{M}$

2.2. Boussinesq-Peregrine type systems. A plethora of Boussinesq systems along with their mechanical balance laws can be derived from the full Euler equations for inviscid and irrotational ideal fluids [47, 13, 2]. In this paper, we consider Peregrine's system [39] which can be derived from the Euler equations, and we make appropriate asymptotic modifications in order to derive systems with favourable properties and overcome certain difficulties arising in Peregrine's systems.

We consider first a modification of Peregrine's system comprised of two decoupled equations for the two components of the depth averaged velocity. This system also generalizes the respective "classical" Boussinesq system of [14] for general bottoms.

In what follows we consider characteristic quantities for typical waves in the Boussinesq regime, in particular a typical wave amplitude $a_{0}$ and length $\lambda_{0}$ and a typical depth $D_{0}$. We will denote the linear wave speed by $c_{0}=\sqrt{g D_{0}}$. We also consider the dimensionless variables

$$
\tilde{\mathbf{x}}=\frac{\mathbf{x}}{\lambda_{0}}, \quad \tilde{t}=\frac{c_{0}}{\lambda_{0}} t, \quad \tilde{\mathbf{u}}=\frac{D_{0}}{a_{0} c_{0}} \mathbf{u}, \quad \tilde{\eta}=\frac{\eta}{a_{0}}, \quad \tilde{D}=\frac{D}{D_{0}} .
$$

Then Peregrine's system (1) can be written in the nondimensional and scaled form:

$$
\begin{aligned}
& \partial_{\tilde{t}} \tilde{\eta}+\nabla \cdot((\tilde{D}+\varepsilon \tilde{\eta}) \tilde{\mathbf{u}})=0, \\
& \partial_{\tilde{t}} \tilde{\mathbf{u}}-\sigma^{2} \frac{1}{2} \tilde{D} \partial_{\tilde{t}} \nabla(\nabla \cdot(\tilde{D} \tilde{\mathbf{u}}))+\sigma^{2} \frac{1}{6} \tilde{D}^{2} \partial_{\tilde{t}} \nabla(\nabla \cdot \tilde{\mathbf{u}})+\varepsilon(\tilde{\mathbf{u}} \cdot \nabla) \tilde{\mathbf{u}}+\nabla \tilde{\eta}=0,
\end{aligned}
$$

where $\varepsilon=a_{0} / D_{0}$ and $\sigma=D_{0} / \lambda_{0}$. In the Boussinesq regime we have $\varepsilon \approx \sigma^{2} \ll 1$ while the Stokes (or Ursel) number is $S=\varepsilon / \sigma^{2}=O(1)$.

The standard derivation of Peregrine's system doesn't take into account the irrotationality condition of the flow, [39], which although is not satisfied exactly, it can be expressed, [34] as:

$$
\tilde{u}_{\tilde{y}}=\tilde{v}_{\tilde{x}}+O\left(\sigma^{2}\right) \text {. }
$$

Using the former relation we observe that the dispersive terms of Peregrine's system can be simplified to the following:

$$
\begin{aligned}
& \nabla\left(\nabla \cdot \tilde{\mathbf{u}}_{\tilde{t}}\right)=\Delta \tilde{\mathbf{u}}_{\tilde{t}}+O\left(\sigma^{2}\right), \\
& \nabla\left(\nabla \cdot\left(\tilde{D} \tilde{\mathbf{u}}_{\tilde{t}}\right)\right)=\tilde{D} \Delta \tilde{\mathbf{u}}_{\tilde{t}}+\nabla\left(\nabla \tilde{D} \cdot \tilde{\mathbf{u}}_{\tilde{t}}\right)+\nabla \tilde{D} \nabla \cdot \tilde{\mathbf{u}}_{\tilde{t}}+O\left(\sigma^{2}\right) .
\end{aligned}
$$

From the second equation of (6) we observe that

$$
\tilde{\mathbf{u}}_{\tilde{t}}=-\nabla \tilde{\eta}+O\left(\varepsilon, \sigma^{2}\right) .
$$

Using (9) into the second equation of (8) we obtain the relation

$$
\nabla\left(\nabla \cdot\left(\tilde{D} \tilde{\mathbf{u}}_{\tilde{t}}\right)\right)=\tilde{D} \Delta \tilde{\mathbf{u}}_{\tilde{t}}-\nabla(\nabla \tilde{D} \cdot \nabla \tilde{\eta})-\nabla \tilde{D} \Delta \tilde{\eta}+O\left(\varepsilon, \sigma^{2}\right)
$$


Furthermore, using (7) we can rewrite the nonlinear term of the second equation of (6) in the following form

$$
(\tilde{\mathbf{u}} \cdot \nabla) \tilde{\mathbf{u}}=\frac{1}{2} \nabla|\tilde{\mathbf{u}}|^{2}+O\left(\sigma^{2}\right) .
$$

Then the Peregrine system can be written, with the help of (8), (10) and (11), in the form:

$$
\begin{aligned}
& \partial_{\tilde{t}} \tilde{\eta}+\nabla \cdot((\tilde{D}+\varepsilon \tilde{\eta}) \tilde{\mathbf{u}})=0, \\
& \partial_{\tilde{t}}\left(\tilde{\mathbf{u}}-\sigma^{2} \frac{1}{3} \tilde{D}^{2} \Delta \tilde{\mathbf{u}}\right)+\frac{1}{2} \sigma^{2} \tilde{D}(\nabla(\nabla \tilde{D} \cdot \nabla \tilde{\eta})+\nabla \tilde{D} \Delta \tilde{\eta})+\varepsilon \frac{1}{2} \nabla|\mathbf{u}|^{2}+\nabla \tilde{\eta}=O\left(\varepsilon^{2}, \sigma^{4}\right) .
\end{aligned}
$$

Discarding the high-order terms, system (12) is written in dimensional form

$$
\begin{aligned}
& \partial_{t} \eta+\nabla \cdot((D+\eta) \mathbf{u})=0 \\
& \partial_{t}\left(\mathbf{u}-\frac{1}{3} D^{2} \Delta \mathbf{u}\right)+\frac{1}{2} g D(\nabla(\nabla D \cdot \nabla \eta)+\nabla D \Delta \eta)+\frac{1}{2} \nabla|\mathbf{u}|^{2}+g \nabla \eta=0 .
\end{aligned}
$$

The last system generalises the classical Boussinesq system derived for horizontal bottom $D(\mathbf{x})=$ $D_{0}$ in [14] to the case of a general bottom. Similar Boussinesq-type systems have been derived in [34]. It is noted that (6) cannot be recovered from the analogous regularized Boussinesq systems derived in [34], even in one-dimensional case by choosing appropriate coefficients, [42].

Assuming that $D$ is very smooth, i.e. $\partial_{x}^{i} D=\partial_{y}^{i} D=O(\varepsilon)$ for $i>0$, we can simplify further system (12) to the following simplified Peregrine system with smooth bottom topography (see also $[18])$, written in dimensional variables

$$
\begin{aligned}
& \partial_{t} \eta+\nabla \cdot((D+\eta) \mathbf{u})=0, \\
& \partial_{t}\left(\mathbf{u}-\frac{1}{3} D^{2} \Delta \mathbf{u}\right)+\frac{1}{2} \nabla|\mathbf{u}|^{2}+g \nabla \eta=0, \quad \text { Simplified Peregrine . }
\end{aligned}
$$

Further, using again the first equation of (8) we get from (12) another system, written in dimensional variables

$$
\begin{aligned}
& \partial_{t} \eta+\nabla \cdot((D+\eta) \mathbf{u})=0 \\
& \partial_{t}\left(\mathbf{u}-\frac{1}{3} D^{2} \nabla(\nabla \cdot \mathbf{u})\right)+\frac{1}{2} \nabla|\mathbf{u}|^{2}+g \nabla \eta=0, \quad \text { Modified Peregrine . }
\end{aligned}
$$

System (15) will be called modified Peregrine system. It is noted that the differences in the dispersive terms between the simplified and the modified systems require different treatment in the boundary conditions as well. The modified and classical Peregrine systems share the same type of elliptic operator while they have different nonlinear terms.

Remark 2.1. Existence and uniqueness of solutions to (1) is studied in [27] in the case where the domain is the whole space. In particular it's been shown that under high-regularity assumptions on the bottom topography $D \in \dot{H}^{N+2}\left(\mathbb{R}^{2}\right)$ for $N \geq 4$, system (1) has a local in time, unique solution $(\mathbf{u}, \eta) \in C\left([0, T], X^{N}\left(\mathbb{R}^{2}\right) \times H^{N}\left(\mathbb{R}^{2}\right)\right)$, where $\dot{H}^{N}\left(\mathbb{R}^{2}\right)=\left\{f \in L_{\text {loc }}^{2}\left(\mathbb{R}^{2}\right), \nabla f \in H^{N-1}\left(\mathbb{R}^{2}\right)\right\}$ and $X^{N}\left(\mathbb{R}^{2}\right)=\left\{\mathbf{u} \in L^{2}\left(\mathbb{R}^{2}\right),\|\mathbf{u}\|_{X^{N}}^{2}=\sum_{|\alpha|=0}^{N}\left\|\partial^{\alpha} \mathbf{u}\right\|_{L^{2}}^{2}+\mu\left\|\partial^{\alpha} \nabla \cdot \mathbf{u}\right\|_{L^{2}}^{2}<\infty\right\}$ for $0<\mu \ll 1$. Wellposedness of system (1) in bounded domains has only been established in one-space dimension, cf. $[41,1]$.

2.3. A new BBM-BBM system. In Peregrine-type systems the mass conservation equation is represented by a first-order partial differential equation. It is known from the theory of finite element method that these kind of differential equations can result to suboptimal convergence rates. The same behaviour is expected in the case of Peregrine's system as well, [7]. For this reason we 
proceed also to the derivation of a BBM-type system with small bottom variations, where the mass conservation will contain a regularisation term.

In [34] a BBM-type system describing weakly nonlinear and weakly dispersive waves propagating over a variable bottom was derived by modifying both the mass and momentum conservation equations so as to avoid the grad - div operator. Here we will perform the same technique but only for the mass equation. We first evaluate the horizontal velocity of the fluid $\mathbf{u}^{\theta}$ at some height $z=-D+\theta(\varepsilon \eta+D)$, with $\theta \in[0,1]$. Then the velocity $\mathbf{u}^{\theta}$ is related to the depth average velocity $\mathbf{u}[36]$ with the formula

$$
\mathbf{u}^{\theta}=\mathbf{u}-\sigma^{2} \tilde{a} \frac{D}{2} \nabla(\nabla \cdot(D \mathbf{u}))-\sigma^{2} \tilde{b} \frac{D^{2}}{3} \nabla(\nabla \cdot \mathbf{u})+O\left(\sigma^{4}\right)
$$

with $\tilde{a}=\theta-1 / 2$ and $\tilde{b}=1 / 2\left[(\theta-1)^{2}-1 / 3\right]$. Substitution of (16) into (6) leads to Nwogu's system

$$
\begin{aligned}
& \eta_{t}+\nabla \cdot\left((D+\varepsilon \eta) \mathbf{u}^{\theta}\right)+\sigma^{2} \nabla \cdot\left[\tilde{a} D^{2} \nabla\left(\nabla \cdot\left(D \mathbf{u}^{\theta}\right)\right)+\tilde{b} D^{3} \nabla\left(\nabla \cdot \mathbf{u}^{\theta}\right)\right]=O\left(\varepsilon \sigma^{2}, \sigma^{4}\right), \\
& \mathbf{u}_{t}^{\theta}+\nabla \eta+\varepsilon\left(\mathbf{u}^{\theta} \cdot \nabla\right) \mathbf{u}^{\theta}+\sigma^{2}\left[\tilde{c} D \nabla\left(\nabla \cdot\left(D \mathbf{u}_{t}^{\theta}\right)\right)+\tilde{d} D^{2} \nabla\left(\nabla \cdot \mathbf{u}_{t}^{\theta}\right)\right]=O\left(\varepsilon \sigma^{2}, \sigma^{4}\right),
\end{aligned}
$$

with

$$
\tilde{a}=\theta-1 / 2, \tilde{b}=1 / 2\left[(\theta-1)^{2}-1 / 3\right], \tilde{c}=\theta-1 \text { and } \tilde{d}=1 / 2(\theta-1)^{2},
$$

(see also $[34,36])$. Following again [34], we have that $D \nabla\left(\nabla \cdot \mathbf{u}^{\theta}\right)=\nabla\left(\nabla \cdot\left(D \mathbf{u}^{\theta}\right)\right)-\nabla\left(\nabla D \cdot \mathbf{u}^{\theta}\right)-$ $\nabla D \nabla \cdot \mathbf{u}^{\theta}$ and substitution into (17) we obtain the regularized system

$\eta_{t}+\nabla \cdot\left((D+\varepsilon \eta) \mathbf{u}^{\theta}\right)-\sigma^{2} \tilde{b} \nabla \cdot\left[D^{2}\left(\nabla\left(\nabla D \cdot \mathbf{u}^{\theta}\right)+\nabla D \nabla \cdot \mathbf{u}^{\theta}\right)\right]+\sigma^{2}(\tilde{a}+\tilde{b}) \nabla \cdot\left[D^{2} \nabla\left(\nabla \cdot\left(D \mathbf{u}^{\theta}\right)\right)\right]=O\left(\varepsilon \sigma^{2}, \sigma^{4}\right)$, $\mathbf{u}_{t}^{\theta}+\nabla \eta+\varepsilon\left(\mathbf{u}^{\theta} \cdot \nabla\right) \mathbf{u}^{\theta}+\sigma^{2}\left[\tilde{c} D \nabla\left(\nabla \cdot\left(D \mathbf{u}_{t}^{\theta}\right)\right)+\tilde{d} D^{2} \nabla\left(\nabla \cdot \mathbf{u}_{t}^{\theta}\right)\right]=O\left(\varepsilon \sigma^{2}, \sigma^{4}\right)$.

Observing now from (19) that $\nabla \cdot\left(D \mathbf{u}^{\theta}\right)=-\eta_{t}+O\left(\varepsilon, \sigma^{2}\right)$ we write Nwogu's system in a new regularized form as

$(20)$

$\eta_{t}+\nabla \cdot\left((D+\varepsilon \eta) \mathbf{u}^{\theta}\right)-\sigma^{2} \tilde{b} \nabla \cdot\left[D^{2}\left(\nabla\left(\nabla D \cdot \mathbf{u}^{\theta}\right)+\nabla D \nabla \cdot \mathbf{u}^{\theta}\right)\right]-\sigma^{2}(\tilde{a}+\tilde{b}) \nabla \cdot\left[D^{2} \nabla \eta_{t}\right]=O\left(\varepsilon \sigma^{2}, \sigma^{4}\right)$, $\mathbf{u}_{t}^{\theta}+\nabla \eta+\varepsilon\left(\mathbf{u}^{\theta} \cdot \nabla\right) \mathbf{u}^{\theta}+\sigma^{2}\left[\tilde{c} D \nabla\left(\nabla \cdot\left(D \mathbf{u}_{t}^{\theta}\right)\right)+\tilde{d} D^{2} \nabla\left(\nabla \cdot \mathbf{u}_{t}^{\theta}\right)\right]=O\left(\varepsilon \sigma^{2}, \sigma^{4}\right)$.

To simplify further the system we make the assumption of small bottom variations. For example we assume that the bottom is very smooth in the sense that the derivatives of $D$ are of $O(\varepsilon)$. Another way to see that is to express $D=D_{0}+D_{b}$, where $D_{b}$ is the variation of the bottom topography from its mean value $D_{0}$, and assume that $D_{b} / D_{0}=O(\varepsilon)$. Although this assumption can result in a system without any contribution of the bottom variations to the dispersive terms, [18], we choose to simplify only the terms $\nabla\left(\nabla D \cdot \mathbf{u}^{\theta}\right)+\nabla D \nabla \cdot \mathbf{u}^{\theta}=O(\varepsilon)$ so as to keep as many bottom effects as possible in the dispersive terms and at the same time derive a simple and easy to handle system. The system (20) is simplified then to

$$
\begin{aligned}
& \eta_{t}+\nabla \cdot\left((D+\varepsilon \eta) \mathbf{u}^{\theta}\right)-\sigma^{2}(\tilde{a}+\tilde{b}) \nabla \cdot\left[D^{2} \nabla \eta_{t}\right]=O\left(\varepsilon \sigma^{2}, \sigma^{4}\right), \\
& \mathbf{u}_{t}^{\theta}+\nabla \eta+\varepsilon\left(\mathbf{u}^{\theta} \cdot \nabla\right) \mathbf{u}^{\theta}+\sigma^{2}\left[\tilde{c} D \nabla\left(\nabla \cdot\left(D \mathbf{u}_{t}^{\theta}\right)\right)+\tilde{d} D^{2} \nabla\left(\nabla \cdot \mathbf{u}_{t}^{\theta}\right)\right]=O\left(\varepsilon \sigma^{2}, \sigma^{4}\right) .
\end{aligned}
$$

This is a BBM-BBM-type system because of the existence of the regularized term in the mass conservation equation. A similar system with additional simplifications to the momentum equations was derived in [34]. It is noted that although we can simplify the dispersive terms in the momentum equation as well, we keep the original form because it is easier to use slip-wall boundary conditions (for example this is harder to do with the relatively more simplified system of [34]) and also to be in agreement with Peregrine's and Nwogu's systems. After dropping the high-order terms, we 
write the system $(21)$ in dimensional form as

$$
\begin{aligned}
& \eta_{t}+\nabla \cdot\left((D+\eta) \mathbf{u}^{\theta}\right)-(\tilde{a}+\tilde{b}) \nabla \cdot\left[D^{2} \nabla \eta_{t}\right]=0, \\
& \mathbf{u}_{t}^{\theta}+g \nabla \eta+\left(\mathbf{u}^{\theta} \cdot \nabla\right) \mathbf{u}^{\theta}+\tilde{c} D \nabla\left(\nabla \cdot\left(D \mathbf{u}_{t}^{\theta}\right)\right)+\tilde{d} D^{2} \nabla\left(\nabla \cdot \mathbf{u}_{t}^{\theta}\right)=0 .
\end{aligned}
$$

In order for the system (22) to be well posed it is required that $1 / 3 \leq \theta^{2} \leq 1$, [13, 14]. Comparing the linear dispersion relation of the system (22) with the corresponding relation of the Euler equations we observe that the parameter $\theta=1$ results to the system which has the closest linear relationship with that of the Euler equations. In order to obtain an exact generalisation of the BBM-BBM system of $[14,13]$ we take $\theta=\sqrt{2 / 3}$ so that $\tilde{a}+\tilde{b}=-(\tilde{c}+\tilde{d})=1 / 6$ and the system after dropping the $\theta$ in the notation is written

$$
\begin{aligned}
& \eta_{t}+\nabla \cdot((D+\eta) \mathbf{u})-\frac{1}{6} \nabla \cdot\left(D^{2} \nabla \eta_{t}\right)=0 \\
& \mathbf{u}_{t}+g \nabla \eta+(\mathbf{u} \cdot \nabla) \mathbf{u}+\left(\sqrt{\frac{2}{3}}-1\right) D \nabla\left(\nabla \cdot\left(D \mathbf{u}_{t}\right)\right)+\left(\frac{5}{6}-\sqrt{\frac{2}{3}}\right) D^{2} \nabla\left(\nabla \cdot \mathbf{u}_{t}\right)=0 .
\end{aligned}
$$

For the rest of the paper we will call (23) BBM-BBM system since it coincides with the well-known one-dimension BBM-BBM system when assuming the bottom is flat [13]. If we take $\theta=\sqrt{1 / 3}$ then we recover the classical Boussinesq system of [13] but with different coefficients in front of the dispersive terms compared to those of the classical Peregrine system when the bottom is not flat. Because the system with $\theta=\sqrt{1 / 3}$ doesn't contribute to the current work, other than the information that in the Boussinesq regime is reasonable to assume that $\nabla D=O(\varepsilon)$, we will not consider it further.

In order to simulate slip wall boundary conditions with the BBM-BBM system we impose zero Neumann boundary conditions $\nabla \eta \cdot \mathbf{n}=0$ on the free-surface elevation and $\mathbf{u} \cdot \mathbf{n}=0$ on the velocity vector field at the boundary of the computational domain. The boundary condition for $\eta$ is not restrictive but is consistent with the total reflection of water waves on the vertical wall, [35], and it is also satisfied by the Euler equations exactly [31]. It also appears naturally into the numerical scheme, since it doesn't require any modification of the finite element spaces (and the Sobolev spaces) from the respective spaces we use for the Peregrine system.

The linear dispersion relations of the previously mentioned systems are given by the relations

$$
\frac{c_{B B M}}{\sqrt{g D}}=\frac{1}{1+(D k)^{2} / 6} \quad \text { and } \quad \frac{c_{\text {Peregrine }}}{\sqrt{g D}}=\frac{1}{\sqrt{1+(D k)^{2} / 3}}
$$

while the linear dispersion relation of the Euler equations is

$$
\frac{c_{\text {Euler }}}{\sqrt{g D}}=\sqrt{\frac{\tanh (D k)}{D k}} .
$$

In the previous relations $D$ is assumed to be the depth of the flat bottom and $k$ is the wavenumber of the linear waves. A comparison of the two dispersion relations of the linearized BBM-BBM and Peregrine systems with those of the linearized Euler equations and Nwogu's system are presented in Figure 2. It is easily observed that Nwogu's system has better dispersive properties compared to other Boussinesq systems (as expected) while Peregrine's system is better than the BBM-BBM system on this account. Of course in the long-wave limit all dispersion relations converge to the correct value and thus we do not expect discrepancies between the solution of the BBM-BBM system and the other Boussinesq systems.

Remark 2.2. Following [34] one can derive also the previously mentioned Boussinesq systems (22) assuming that the bottom depends on time, i.e. it is of the form $D(x, y)+\zeta(x, y, t)$, where the timedependent part is of the same order of magnitude as the free-surface elevation $\eta$. For example the 


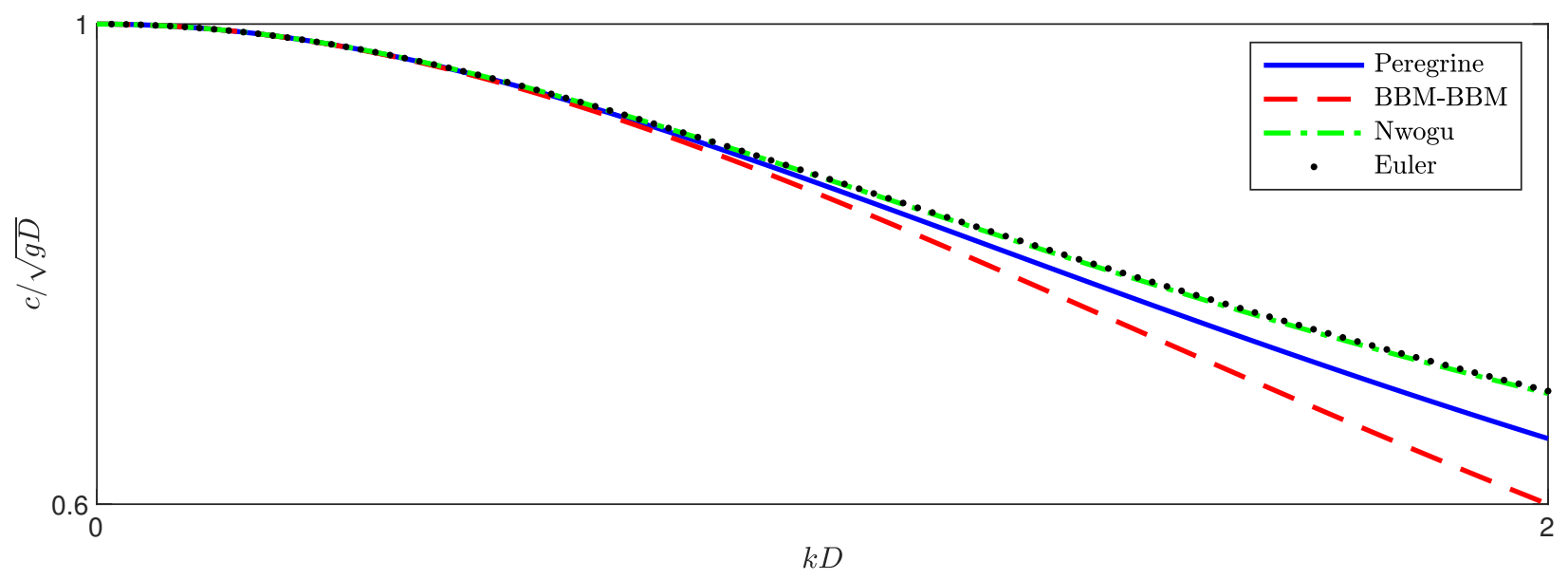

FiguRE 2. Comparison of linear dispersion relations

new BBM-BBM system takes the form:

$$
\begin{aligned}
& \eta_{t}+\nabla \cdot\left((D+\zeta+\eta) \mathbf{u}^{\theta}\right)-(\tilde{a}+\tilde{b}) \nabla \cdot\left[D^{2} \nabla \eta_{t}\right]=-\tilde{a} \nabla \cdot\left(D^{2} \nabla \zeta_{t}\right)-\zeta_{t}, \\
& \mathbf{u}_{t}^{\theta}+g \nabla \eta+\left(\mathbf{u}^{\theta} \cdot \nabla\right) \mathbf{u}^{\theta}+\tilde{c} D \nabla\left(\nabla \cdot\left(D \mathbf{u}_{t}^{\theta}\right)\right)+\tilde{d} D^{2} \nabla\left(\nabla \cdot \mathbf{u}_{t}^{\theta}\right)=-\tilde{c} D \nabla \zeta_{t t},
\end{aligned}
$$

where $\tilde{a}, \tilde{b}, \tilde{c}$ and $\tilde{d}$ are given by (18). The corresponding Peregrine system (1) can be written as

$$
\begin{aligned}
& \eta_{t}+\nabla \cdot[(D+\zeta+\eta) \mathbf{u}]=-\zeta_{t}, \\
& \mathbf{u}_{t}+g \nabla \eta+(\mathbf{u} \cdot \nabla \cdot) \mathbf{u}-\frac{1}{2} D \nabla\left(\nabla \cdot\left(D \mathbf{u}_{t}\right)\right)+\frac{1}{6} D^{2} \nabla\left(\nabla \cdot \mathbf{u}_{t}\right)=\frac{D}{2} \nabla \zeta_{t t} .
\end{aligned}
$$

The forcing terms of the moving bottom terms can be used not only for simulating waves generated by moving bottoms such as tsunamis [34] but also for the accurate simulation of waves generated due to a wavemaker. We discuss this application in detail in a later section (see also [44, 45]).

All the aforementioned Boussinesq-Peregrine systems are augmented with initial and boundary conditions and can be written in the following compact form, where the values of $\mathcal{E}_{s}, \mathcal{E}$ and $\mathcal{N}$ are presented in Table 1

$$
\begin{aligned}
\partial_{t} \mathcal{E}_{s}(\eta)+\nabla \cdot((D+\eta) \mathbf{u}) & =0 \\
\partial_{t} \mathcal{E}(\mathbf{u})+\mathcal{N}(\mathbf{u})+g \nabla \eta & =0 \\
\mathbf{u} \cdot \mathbf{n} & =0, \\
\mathbf{u} & =0, \text { on } \partial \Omega \text { on } \partial \Omega \text { Simplified, } \\
\nabla \eta \cdot \mathbf{n} & =0, \text { on } \partial \Omega \text { BBM-BBM, } \\
\mathbf{u}(\mathbf{x}, 0)=\mathbf{u}_{0}(\mathbf{x}), \eta(\mathbf{x}, 0) & =\eta_{0}(\mathbf{x})
\end{aligned}
$$

\section{Finite element approximations}

We consider now the application of the finite element method for obtaining approximations to the models presented in the previous section. To simplify the presentation of the finite element method we assume in the sequel, without loss of generality, that $\Omega$ is a closed polygonal domain that might contain holes and with boundary $\Gamma$. On $\Omega$ we consider a regular triangulation $\mathcal{T}_{h}$, [19] consisting of triangles $K, \bar{\Omega}=\cup_{K} K$, with $h_{K}$ denoting the diameter of $K$ and $h$ the maximum 
TABlE 1. Peregrine-Boussinesq type models

\begin{tabular}{||c||c||c||c||}
\hline Model & $\mathcal{E}_{s}(\eta)$ & $\mathcal{E}(\mathbf{u})$ & $\mathcal{N}(\mathbf{u})$ \\
\hline \hline Classical & $\eta$ & $\mathbf{u}-\frac{1}{2} D \nabla(\nabla \cdot(D \mathbf{u}))+\frac{1}{6} D^{2} \nabla(\nabla \cdot \mathbf{u})$ & $(\mathbf{u} \cdot \nabla) \mathbf{u}$ \\
\hline Simplified & $\eta$ & $\mathbf{u}-\frac{1}{3} D^{2} \Delta \mathbf{u}$ & $\frac{1}{2} \nabla|\mathbf{u}|^{2}$ \\
\hline Modified & $\eta$ & $\mathbf{u}-\frac{1}{3} D^{2} \nabla(\nabla \cdot \mathbf{u})$ & $\frac{1}{2} \nabla|\mathbf{u}|^{2}$ \\
\hline BBM-BBM & $\eta-\frac{1}{6} \nabla \cdot\left(D^{2} \nabla \eta\right)$ & $\mathbf{u}+\left(\sqrt{\frac{2}{3}}-1\right) D \nabla(\nabla \cdot(D \mathbf{u}))+\left(\frac{5}{6}-\sqrt{\frac{2}{3}}\right) D^{2} \nabla(\nabla \cdot \mathbf{u})$ & $(\mathbf{u} \cdot \nabla) \mathbf{u}$ \\
\hline
\end{tabular}

diameter of these triangles, $h=\max \left\{h_{K}, K \in \mathcal{T}_{h}\right\}$. Further, for a positive integer $r$ let $P^{r}(K)$ denotes the space of bivariate polynomials of degree $\leq r$ on $K$.

For the approximation of $(\eta, \mathbf{u})$ we consider the standard finite element spaces $V_{h}^{r}$ consisting of Lagrange-type polynomial basis functions of degree at most $r$ :

$$
V_{h}^{r}=\left\{\phi \in C(\Omega):\left.\phi\right|_{K} \in P^{r}(K), K \in \mathcal{T}_{h}\right\}, \quad U_{h}^{r}=V_{h}^{r} \times V_{h}^{r},
$$

and $V_{h}^{r}$ and $U_{h}^{r}$ are finite dimensional subspaces of $V$ and $V \times V$ respectively.

Remark 3.1. The form of the elliptic part of the Peregrine system (1) suggests of using finite element subspaces of $H($ div,$\Omega)$. This approach can be worked out possibly in the framework of a mixed finite element method where an appropriate discretization of the nonlinear term in (1) must be considered. Here, we follow a different approach and we consider finite element subspaces of $H^{1}(\Omega)$.

Remark 3.2. In general we will seek approximations of $\eta$ and $\mathbf{u}$ from different finite element subspaces $V_{h}^{r_{1}}$ and $U_{h}^{r_{2}}$ respectively, where $r_{1}, r_{2}$ are, possibly different, positive integers.

Remark 3.3. The numerical results reported in this and the following sections were obtained by using in part the FEniCS computational framework, [3, 33].

3.1. Fully discrete schemes. We consider now the finite element discretization of systems (26). For $t>0$, we seek $\left(\eta_{h}(t), \mathbf{u}_{h}(t)\right) \in V_{h}^{r_{1}} \times U_{h}^{r_{2}}$ such that

$$
\begin{array}{ll}
\left(\partial_{t} \mathcal{E}_{s}\left(\eta_{h}\right), \psi\right)-F_{s}\left(\eta_{h}, \mathbf{u}_{h}, \psi\right)=0, & F_{s}\left(\eta_{h}, \mathbf{u}_{h}, \psi\right)=-\left(\nabla \cdot\left(\left(D+\eta_{h}\right) \mathbf{u}_{h}\right), \psi\right), \quad \forall \psi \in V_{h}^{r_{1}}, \\
\left(\partial_{t} \mathcal{E}\left(\mathbf{u}_{h}\right), \boldsymbol{\phi}\right)-F_{u}\left(\eta_{h}, \mathbf{u}_{h}, \boldsymbol{\phi}\right)=0, \quad F_{u}\left(\eta_{h}, \mathbf{u}_{h}, \boldsymbol{\phi}\right)=-\left(\mathcal{N}\left(\mathbf{u}_{h}\right), \boldsymbol{\phi}\right)-g\left(\nabla \eta_{h}, \boldsymbol{\phi}\right), \quad \forall \boldsymbol{\phi} \in U_{h}^{r_{2}} .
\end{array}
$$

Performing a standard integration by parts, as in the elliptic case (30) we obtain the following semidiscrete formulation

$$
\begin{aligned}
\partial_{t} \mathcal{C}_{s}\left(\eta_{h}, \psi\right)=F_{s}\left(\eta_{h}, \mathbf{u}_{h}, \psi\right), & \forall \psi \in V_{h}^{r_{1}}, \\
\partial_{t} \mathcal{C}\left(\mathbf{u}_{h}, \boldsymbol{\phi}\right)=F_{u}\left(\eta_{h}, \mathbf{u}_{h}, \boldsymbol{\phi}\right), & \forall \boldsymbol{\phi} \in U_{h}^{r_{2}}, \\
\eta_{h}(0)=\mathcal{P} \eta_{0}, \mathbf{u}_{h}(0)=\mathcal{P} \mathbf{u}_{0}, &
\end{aligned}
$$


where

$$
\begin{aligned}
\mathcal{C}\left(\mathbf{u}_{h}, \boldsymbol{\phi}\right):=\mathcal{A}\left(\mathbf{u}_{h}, \boldsymbol{\phi}\right)+ & \mathcal{B}\left(\mathbf{u}_{h}, \boldsymbol{\phi}\right)+\frac{1}{3} \frac{C_{N}}{h}\left\langle D \mathbf{u}_{h} \cdot \mathbf{n}, D \boldsymbol{\phi} \cdot \mathbf{n}\right\rangle=(\mathbf{f}, \boldsymbol{\phi}), \quad \forall \boldsymbol{\phi} \in U_{h}^{r}, \\
\mathcal{A}(\mathbf{u}, \mathbf{v})= & (\mathbf{u}, \mathbf{v})+\frac{1}{3}(\nabla \cdot(D \mathbf{u}), \nabla \cdot(D \mathbf{v}))+\frac{1}{6}(\mathbf{u} \cdot \nabla D, \mathbf{v} \cdot \nabla D) \\
& +\frac{1}{6}(\mathbf{u} \cdot \nabla D, \nabla \cdot(D \mathbf{v}))-\frac{1}{6}(\nabla \cdot(D \mathbf{u}), \mathbf{v} \cdot \nabla D) \\
\mathcal{B}\left(\mathbf{u}_{h}, \boldsymbol{\phi}\right)= & -\frac{1}{3}\left\langle\nabla \cdot\left(D \mathbf{u}_{h}\right), D \boldsymbol{\phi} \cdot \mathbf{n}\right\rangle-\frac{1}{6}\left\langle\mathbf{u}_{h} \cdot \nabla D, D \boldsymbol{\phi} \cdot \mathbf{n}\right\rangle \\
& -\frac{1}{3}\left\langle\nabla \cdot(D \boldsymbol{\phi}), D \mathbf{u}_{h} \cdot \mathbf{n}\right\rangle-\frac{1}{6}\left\langle\boldsymbol{\phi} \cdot \nabla D, D \mathbf{u}_{h} \cdot \mathbf{n}\right\rangle
\end{aligned}
$$

and $\mathcal{C}_{s}\left(\eta_{h}, \psi\right)=\left(\eta_{h}, \psi\right)$ or $\mathcal{C}_{s}\left(\eta_{h}, \psi\right)=\left(\eta_{h}, \psi\right)+\left(\nabla \eta_{h}, \nabla \psi\right)$ while $\mathcal{P}$ denotes the $L^{2}$-projection on the finite element space. The bilinear forms $\mathcal{C}(\cdot, \cdot)$ and $\mathcal{C}_{s}(\cdot, \cdot)$ are continuous and coercive with respect to $\|D \cdot\|_{\mathrm{H}(\mathrm{div})}$ and $\|\cdot\|_{1}$ respectively, thus system (29) has a unique solution.

The system of ordinary differential equations (29) is discretized by using the classical fourthorder accurate, four-stage explicit Runge-Kutta method(RK4). Indeed, this method is used as a time stepping mechanism for all the numerical experiments presented in the sequel. The method is described by the following Butcher tableau,

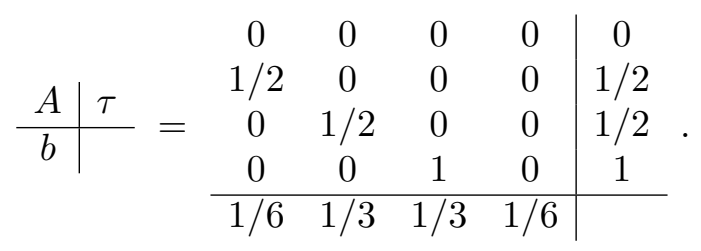

We use a uniform time-step $\Delta t$ and the temporal grid is $t^{n}=n \Delta t$, where $n=0,1, \cdots$. Given the ODE $y^{\prime}=\Phi(t, y)$, one step of this four-stage RK scheme (with $y^{n}$ approximating $y\left(t^{n}\right)$ ) is:

$$
\begin{aligned}
& \text { for } i=1 \rightarrow 4 \text { do } \\
& \quad \tilde{y}^{n, i}=y^{n}+\Delta t \sum_{j=1}^{i-1} a_{i j} y^{n, j} \\
& \quad y^{n, i}=\Phi\left(t^{n, i}, \tilde{y}^{n, i}\right), \quad t^{n, i}=t^{n}+\tau_{i} \Delta t \\
& \text { end for } \\
& y^{n+1}=y^{n}+\Delta t \sum_{i=1}^{4} b_{i} y^{n, i} .
\end{aligned}
$$

We introduce the notation $\left(H^{n}, \mathbf{U}^{n}\right) \approx\left(\eta_{h}\left(t^{n}\right), \mathbf{u}_{h}\left(t^{n}\right)\right)$ and $\left(\mathcal{D} H^{k}, \mathcal{D} \mathbf{U}^{k}\right)=\left(H^{k}-H^{n}, \mathbf{U}^{k}-\mathbf{U}^{n}\right)$. Then, one complete timestep of the RK4 method applied to system (29) is

$$
\begin{aligned}
& \text { for } i=1 \rightarrow 4 \text { do } \\
& \mathcal{D} \tilde{H}^{n, i}=\Delta t \sum_{j=1}^{i-1} a_{i j} H^{n, j} \\
& \mathcal{D} \tilde{\mathbf{U}}^{n, i}=\Delta t \sum_{j=1}^{i-1} a_{i j} \mathbf{U}^{n, j} \\
& \mathcal{C}_{s}\left(H^{n, i}, \psi\right)=F_{s}\left(t^{n, i}, \tilde{H}^{n, i}, \tilde{\mathbf{U}}^{n, i}, \psi\right), \quad t^{n, i}=t^{n}+\tau_{i} \Delta t \\
& \mathcal{C}\left(\mathbf{U}^{n, i}, \boldsymbol{\phi}\right)=F_{u}\left(t^{n, i}, \tilde{H}^{n, i}, \tilde{\mathbf{U}}^{n, i}, \boldsymbol{\phi}\right), \quad t^{n, i}=t^{n}+\tau_{i} \Delta t \\
& \text { end for } \\
& \mathcal{D} H^{n+1}=\Delta t \sum_{i=1}^{4} b_{i} H^{n, i}, \mathcal{D} \mathbf{U}^{n+1}=\Delta t \sum_{i=1}^{4} b_{i} \mathbf{U}^{n, i} .
\end{aligned}
$$

It is easily seen that at every timestep $\Delta t$ it is required the numerical solution of four linear systems with the time-independent matrix $\mathcal{C}_{s}\left(\psi_{i}, \psi_{j}\right)$ and four with the matrix $\mathcal{C}\left(\phi_{i}, \boldsymbol{\phi}_{j}\right)$. In our computer code we used the sparse $L U$ decomposition of FEniCS, which appeared to be the most efficient method compared to other iterative methods such as the conjugate gradient (with the Jacobi preconditioner) and the generalised minimal residual methods (with the incomplete $L U$ preconditioner). 
3.2. Spatial convergence rates and stability. We turn now our attention to the convergence of the fully-discrete scheme. We begin with the validation of the scheme by performing accuracy tests and computing the experimental order of convergence $(E O C)$ for various choices of the finite element spaces. We compute the EOC based on the error between the exact and approximate solution measured in the $L^{2}, H^{1}$ norms:

$$
E O C=\log \left(\frac{E_{1}}{E_{2}}\right) / \log \left(\frac{h_{1}}{h_{2}}\right),
$$

where $E_{1}, E_{2}$ are the normed errors and $h_{1}, h_{2}$ are the corresponding mesh sizes.

It is known that the standard Galerkin method applied to Peregrine's system in 1D with reflective boundary conditions converges with a suboptimal convergence rate, $[6,7]$. Specifically, it was shown that for linear elements the convergence rates of the $L^{2}$ error for the velocity $\mathbf{u}$ was optimal 2, while for the free surface $\eta$ was suboptimal and equal to $3 / 2$. The convergence rates of the $H^{1}$ error was also shown to decrease with a suboptimal rate of $1 / 2$ for the free-surface variable $\eta$, while the convergence rate of the respective $H^{1}$ error for the velocity variable was optimal and equal to 1 .

In similar situations it has been observed that using different finite element spaces for the dependent variables can be beneficial and can result to a fully-discrete scheme that converges with optimal rate to the exact solution, $[8,35]$. On the other hand, in the case of other Boussinesq-type systems, such as the Bona-Smith system, the order of convergence can be different between one and two space dimensions, $[24,9]$. For these reasons, it's unrealistic to have expectations for specific convergence rates for our numerical method unless we check.

We compute the EOC's by the method of manufactured solutions. We choose an exact solution $(\eta, \mathbf{u})$, we compute appropriate right-hand side $\mathbf{f}$ using the differential equations and then solve a non-homogeneous problem. For the classical, modified and the new Peregrine systems we choose an exact solution that satisfies the boundary condition $\mathbf{u} \cdot \mathbf{n}=0$. However for the simplified Boussinesq system we need to choose a different exact solution since an extra boundary condition $\mathbf{u}=0$ is needed due to the presence of the Laplacian in the reguarisation operator. As for $\eta$, Neumann boundary condition is taken for the BBM-BBM system due to the presence of the BBM-term in the continuity equation, while no extra boundary condition is considered for the other models. The value of $\eta$ is the same for all systems. The exact values for $(\eta, \mathbf{u})$ are :

$$
\begin{array}{ll}
\mathbf{u}(\mathbf{x}, t)=e^{(x+y) t}\left(\cos \left(\frac{\pi}{2} y\right) \sin (\pi x), \cos \left(\frac{\pi}{2} x\right) \sin (\pi y)\right), & \text { Classical, Modified, BBM-BBM }, \\
\mathbf{u}(\mathbf{x}, t)=e^{t}\left(x \cos \left(\frac{\pi}{2} x\right) \sin (\pi y), y \cos \left(\frac{\pi}{2} y\right) \sin (\pi x)\right), & \text { Simplified } \\
\eta(\mathbf{x}, t)=e^{t} \cos (\pi x) \sin (\pi y) .
\end{array}
$$

We also consider a linearly varying bottom topography

$$
H(x, y)=-\frac{1}{20}(x+y)+\frac{3}{2},
$$

which represents a sloping bottom with slope $1 / 20$. We integrate numerically the non-homogeneous Peregrine-Boussinesq type systems and we compute the experimental orders of convergence $(E O C)$ based on the $L^{2}, H^{1}$ and $\mathrm{H}$ (div) errors between the numerical and the exact solutions, as in (32). All the computations where performed in the unit square $\Omega=[0,1] \times[0,1]$ with final time $T=1$. On $\Omega$ we consider a regular uniform triangulation $\mathcal{T}_{h}$ consisting of equilateral triangles and we denote as $h$ the length of a side. The temporal time step was taken $\Delta t=5 \times 10^{-4}$ to ensure that the contribution of the time-stepping method to the total error is negligible. For approximation we use finite element spaces $V_{h}^{r_{1}}, U_{h}^{r_{2}}$ with various polynomial degrees $r_{1}, r_{2}=1,2,3,4$.

The errors measured in $L^{2}$ and $H^{1}$ norms for $(\eta, \mathbf{u})$ are shown in Figures 3 and 4 respectively for five different combinations of the values $r_{1}$ and $r_{2}$. The behaviour of the error for each variable 
and each model is presented with solid lines in log-log scale for various values of the discretization parameter $h$. In each graph the corresponding optimal rate is depicted by a dashed line with optimal slope.
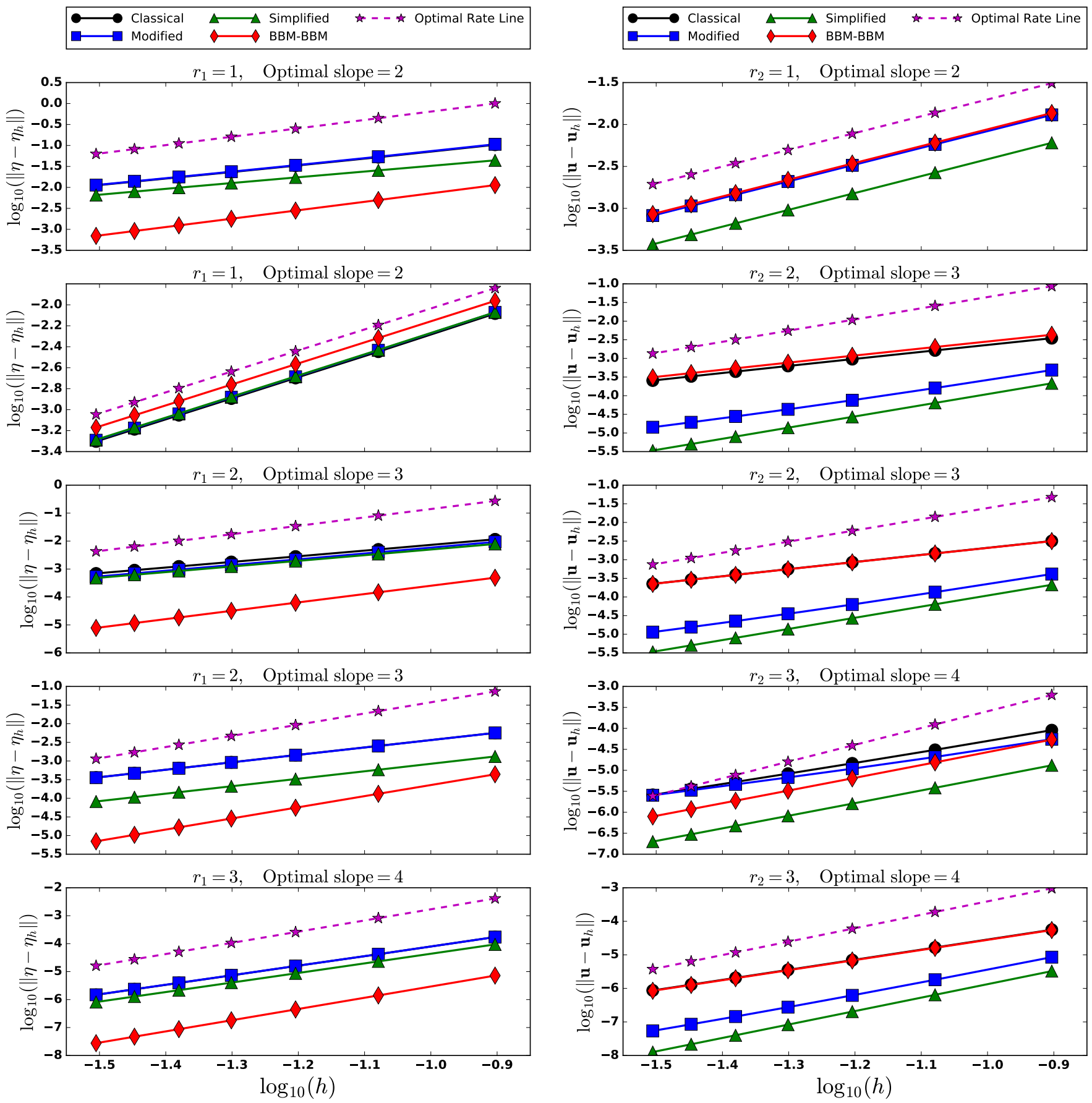

Figure 3. Log-Log plot of errors in the $L^{2}$-norm : $\eta$ in left column and $\mathbf{u}$ in right column.

We consider first $\left(\eta_{h}, \mathbf{u}_{h}\right)$ in finite element spaces $\left(V_{h}^{r}, U_{h}^{r}\right)$ of the same polynomial degree $r$ with $r=1,2,3,4$. The EOC's for $\eta$ in the $L^{2}$ and $H^{1}$ norms for the classical, modified and 

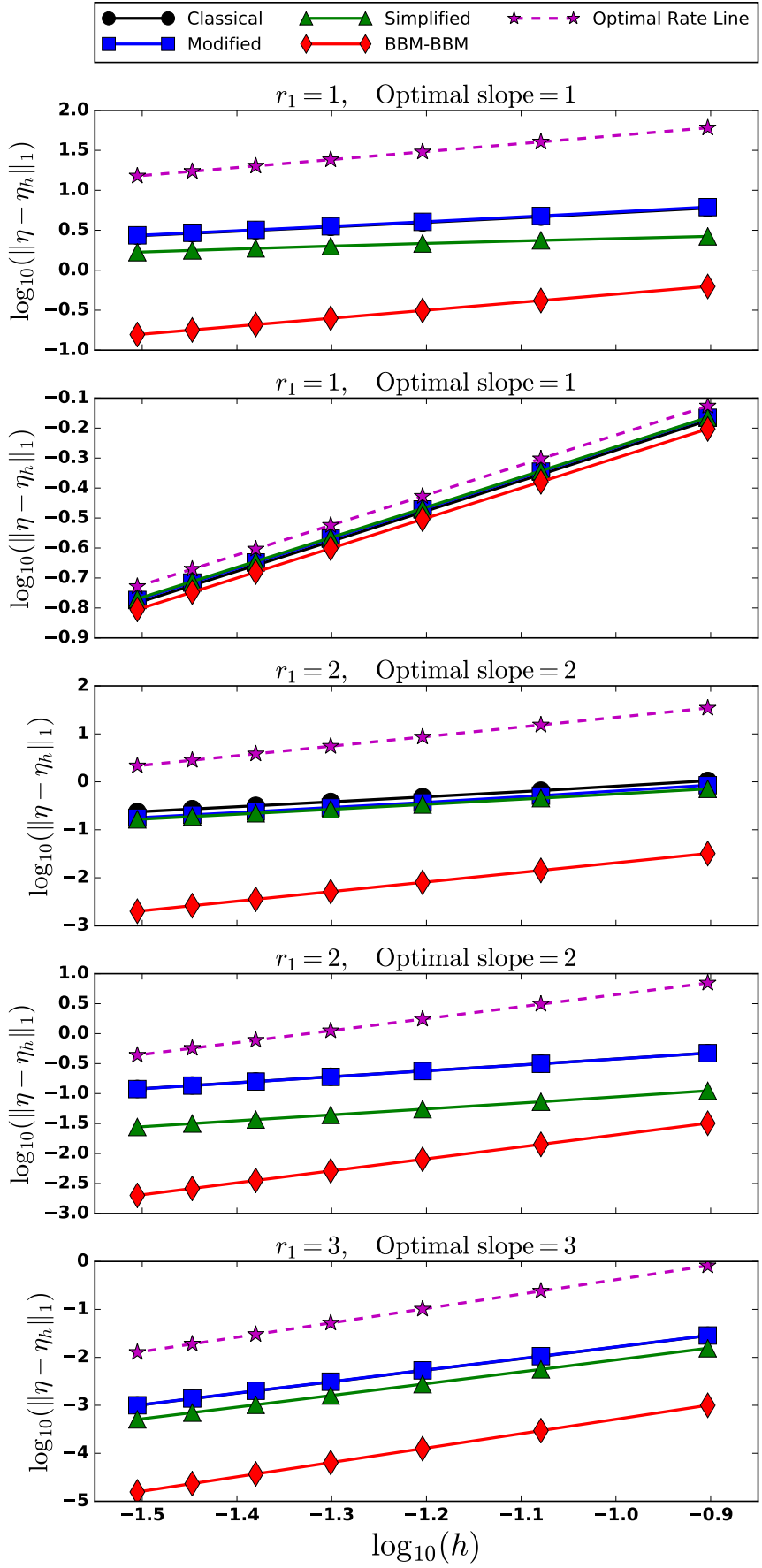
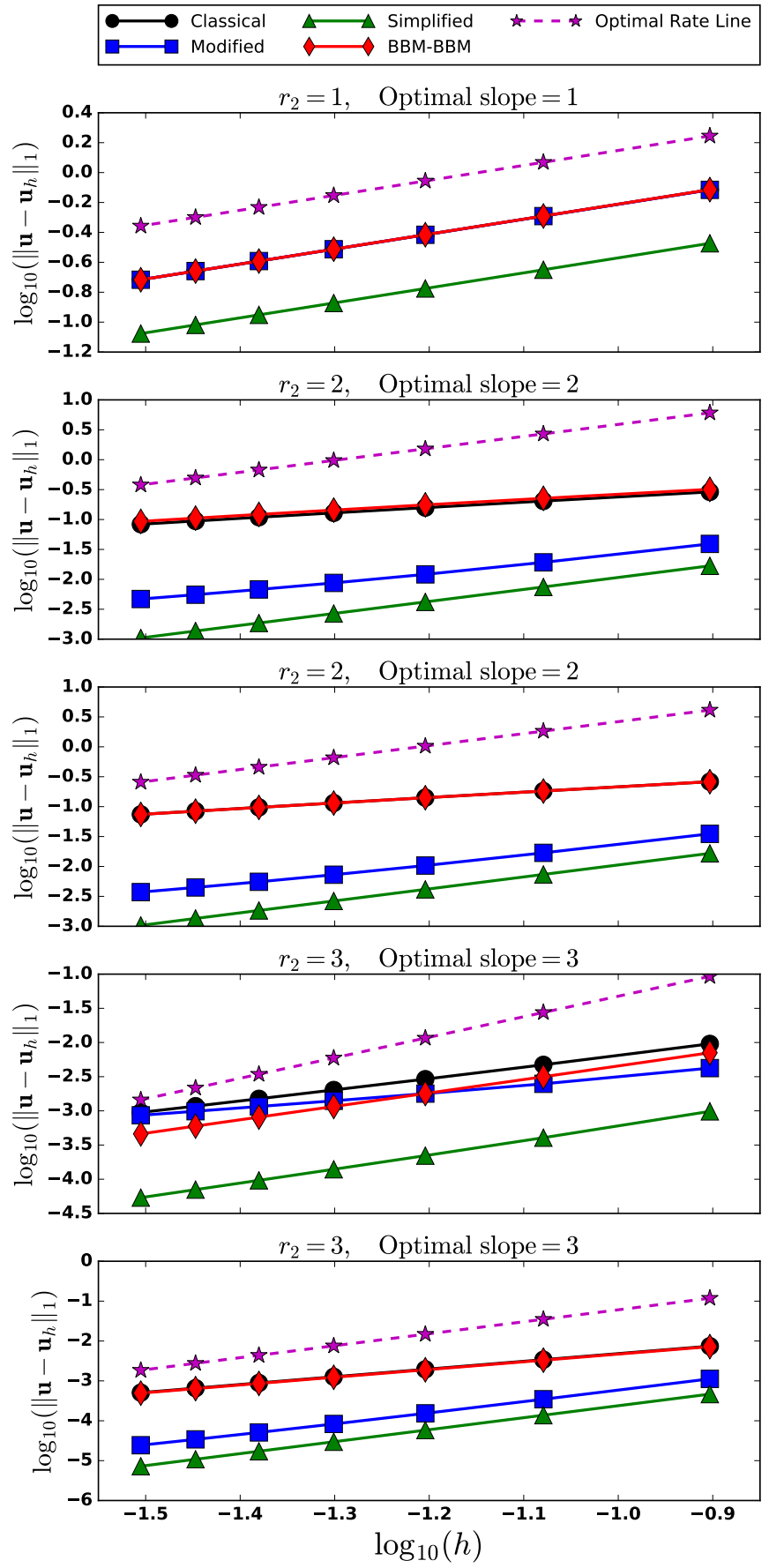

Figure 4. Log-Log plot of errors in the $H^{1}$-norm : $\eta$ in left column and $\mathbf{u}$ in right column.

simplified systems are exactly the same for all values of $r$, but suboptimal by $1 / 2$ for the odd degree polynomials and suboptimal by 1 for the even degree basis functions. For the $B B M-B B M$ system the optimal EOC is obtained in both norms. The latter is attributed to the presence of a BBM-type term in the continuity equation, first equation of (23). The situation is somehow different for the EOC's for $\mathbf{u}$. For $r=1$ the EOC's are optimal in the $L^{2}$ and $H^{1}$ norms for all systems. However for $r=2,3,4$ the situation differs and for the classical, modified and BBM-BBM 
systems the EOC's in both $L^{2}$ and $H^{1}$ norms are suboptimal by 1 . On the other hand, the optimal EOC is achieved for the simplified system since the corresponding elliptic operator involves the Laplacian.

We tested also three cases where the degree of polynomials $\left(r_{1}, r_{2}\right)$ of the finite element spaces $\left(V_{h}^{r_{1}}, U_{h}^{r_{2}}\right)$ differ by 1 and in particular we took $\left(r_{1}, r_{2}\right)=(1,2),(2,3),(3,4)$. For odd values of $r_{1}$ the optimal EOC's for $\eta$ were obtained in both norms and for all systems, while for even values of $r_{1}$ all the EOC's are suboptimal by 1, except for the BBM-BBM system which the EOC's are optimal. For $\mathbf{u}$ and for odd values of $r_{2}$ the resulting EOC's are suboptimal by 1 in both norms for the classical, modified and BBM-BBM systems, but optimal for the simplified system. For even values of $r_{2}$ the convergence rates for $\mathbf{u}$ are suboptimal by 1 in both norms for the simplified, BBM-BBM systems and suboptimal by 2 for the classical and modified systems. Convergence rates for $\mathbf{u}$ were also computed in the $\mathrm{H}$ (div)-norm. These rates proven to be optimal for all the systems and all the choices of $r_{1}$ and $r_{2}$.

All these results are summarized in Table 2 where by $E_{k}(\cdot), k=0,1$ we denoted the EOC in the $L^{2}$ and $H^{1}$ respectively, while $E_{d}(\cdot)$ is the corresponding EOC in the $\mathrm{H}(\mathrm{div})$ norm.

TABLE 2. EOC's for $(\eta, \mathbf{u})$ for various choices of polynomial degree $r_{1}, r_{2}$

\begin{tabular}{|c|c|c|c|c|c|c|c|c|c|c|}
\hline & \multicolumn{5}{|c|}{ Classical } & \multicolumn{5}{|c|}{ Modified } \\
\hline & \multicolumn{2}{|c|}{$\eta$} & \multicolumn{3}{|c|}{$\mathbf{u}$} & \multicolumn{2}{|c|}{$\eta$} & \multicolumn{3}{|c|}{$\mathbf{u}$} \\
\hline$\left(r_{1}, r_{2}\right)$ & $E_{0}(\eta)$ & $E_{1}(\eta)$ & $E_{0}(\mathbf{u})$ & $E_{1}(\mathbf{u})$ & $E_{d}(\mathbf{u})$ & $E_{0}(\eta)$ & $E_{1}(\eta)$ & $E_{0}(\mathbf{u})$ & $E_{1}(\mathbf{u})$ & $E_{d}(\mathbf{u})$ \\
\hline$(1,1)$ & 1.5 & 0.5 & 2 & 1 & 1 & 1.5 & 0.5 & 2 & 1 & 1 \\
\hline$(2,2)$ & 2 & 1 & 2 & 1 & 2 & 2 & 1 & 2 & 1 & 2 \\
\hline$(3,3)$ & 3.5 & 2.5 & 3 & 2 & 3 & 3.5 & 2.5 & 3 & 2 & 3 \\
\hline$(4,4)$ & 4 & 3 & 4 & 3 & 4 & 4 & 3 & 4 & 3 & 4 \\
\hline$(1,2)$ & 2 & 1 & 2 & 1 & 2 & 2 & 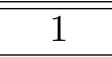 & 2 & 1 & 2 \\
\hline$(2,3)$ & 2.5 & 1 & 2 & 1.5 & 2 & 2 & 1 & 2 & 1 & 3 \\
\hline \multirow[t]{3}{*}{$(3,4)$} & 4 & 3 & 4 & 3 & 4 & 4 & 3 & 4 & 3 & 4 \\
\hline & \multicolumn{5}{|c|}{ Simplified } & \multicolumn{5}{|c|}{ BBM-BBM } \\
\hline & \multicolumn{2}{|c|}{$\eta$} & \multicolumn{3}{|c|}{$\mathbf{u}$} & \multicolumn{2}{|c|}{$\eta$} & \multicolumn{3}{|c|}{$\mathbf{u}$} \\
\hline$\left(r_{1}, r_{2}\right)$ & $E_{0}(\eta)$ & $E_{1}(\eta)$ & $E_{0}(\mathbf{u})$ & $E_{1}(\mathbf{u})$ & $E_{d}(\mathbf{u})$ & $E_{0}(\eta)$ & $E_{1}(\eta)$ & $E_{0}(\mathbf{u})$ & $E_{1}(\mathbf{u})$ & $E_{d}(\mathbf{u})$ \\
\hline$(1,1)$ & 1.5 & 0.5 & 2 & 1 & 1 & 2 & 1 & 2 & 1 & 1 \\
\hline$(2,2)$ & 2 & 1 & 3 & 2 & 2 & 3 & 2 & 2 & 1 & 2 \\
\hline$(3,3)$ & 3.5 & 2.5 & 4 & 3 & 3 & 4 & 3 & 3 & 2 & 3 \\
\hline$(4,4)$ & 4 & 3 & 5 & 4 & 4 & 5 & 4 & 4 & 3 & 4 \\
\hline 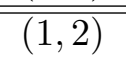 & 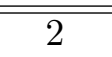 & $\overline{1} 1$ & 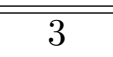 & 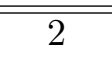 & 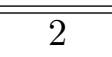 & 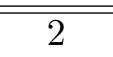 & $\bar{~} 1$ & 2 & $\bar{~} 1$ & 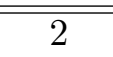 \\
\hline$(2,3)$ & 2 & 1 & 3 & 2 & 3 & 3 & 2 & 3 & 2 & 3 \\
\hline$(3,4)$ & 4 & 3 & 5 & 4 & 4 & 4 & 3 & 4 & 3 & 4 \\
\hline
\end{tabular}

Remark 3.4. The computational convergence rates are the same as in the one-dimensional case, [35], which suggests the same behaviour of the system in two-dimensions.

The presence of the incomplete-elliptic operator $\mathcal{E}(\mathbf{u})$, Table $1(\mathrm{a})$ in the left hand side of the systems (26) makes the corresponding o.d.e systems (29) mildly stiff and the fully discrete schemes are stable under a relaxed CFL-type condition. We provide now some indicative numerical results concerning the size of the Courant number. To do so, we consider a solitary wave of various amplitudes $A$ and we study its propagation using the classical Peregrine system (1) and the newly derived BBM-BBM system (23). For both systems we compute the largest Courant number such 
that the propagation is stable. We consider a channel $[-50,100]$ of constant $\operatorname{depth} D(\mathbf{x})=1$ and the initial location of the solitary wave is at $x=-20$. The channel is covered by an unstructured triangulation of 33192 triangles with a minimum size $h_{\min } \sim 0.09$. We integrate both systems up to $T=100$ and we compute Courant number defined as $c_{s} \frac{\Delta t}{h_{\min }}$, where $c_{s}=\sqrt{g(D+A)}$ is the phase speed of the solitary wave and $\Delta t$ is the time step. We tested solitary waves with five different amplitudes, namely we took $A=0.05,0.1,0.2,0.4,0.5$. The maximum allowed Courant numbers in all test cases were of the order of 10 showing that the method is essentially unconditionally stable, [8].

Remark 3.5. The suboptimal convergence rates for the velocity field in the $L^{2}$-norm appeared for the classical, modified Peregrine and BBM-BBM systems are due to the combination of variable bathymetry and the Nitsche method for the slip-wall boundary conditions. When flat bottom topography is considered, the convergence rates for the velocity field are optimal in the $L^{2}$-norm in all these cases.

\section{Solitary Waves}

In this section we study the accuracy of the numerical methods in the propagation of a solitary wave and we present some differences in the behaviour of the numerical solutions between the Peregrine and BBM-BBM systems. Since both systems with flat bottom are reduced to the respective Boussinesq systems of [13] it is known that they possess classical line solitary wave solution [23] that propagate with constant speed and without change in shape at any direction. Due to the lack of exact analytical formulas for the solitary wave solutions of the systems at hand we employed the Petviashvili method for their numerical generation. The method is presented briefly in the Appendix A.

4.1. Solitary wave propagation. The finite element method has been applied to the Peregrine system before using linear elements in several works. While in $[5,10,43]$ the propagation of small amplitude solitary waves has been reported to be approximated in a satisfactory way using linear elements, in [48] was shown that significant oscillations can be generated behind large amplitude solitary waves during their propagation along a channel. These large oscillations do not affect the stability of the numerical method but they pollute the numerical solution and decrease its resolution. The streamline diffusion method was proposed in [48], to smooth-out the trailing spurious oscillations. However, the streamline diffusion method is expensive, hard to implement and also adds unwanted damping to the solution. Studying the propagation of a solitary wave of amplitude $A=0.3$ of Peregrine's system in the channel $[-20,30] \times[-1,1]$ with depth $D=1$ up to time $T=25$ we observed that using linear elements for all the dependent variables leads to the generation of significant oscillations behind the solitary pulse as shown in Figure 5 (a), see also Figure 6. These oscillations are of the exact same nature with those reported in [48]. Repeating the same experiment with the BBM-BBM system for a solitary wave of the same amplitude and with the same elements we didn't observe spurious oscillations as the inversion of the elliptic operator in the mass conservation equation regularizes the solution completely. The regularized solution of the BBM-BBM system is presented in Figure 5 (b). Increasing also the degree of the finite elements for the approximation of the velocity field $\mathbf{u}$ we observe that the spurious oscillations are eliminated. Of course, because the initial conditions are not exact traveling waves to the numerical model but just approximations, it is expected that the solitary pulse will be followed by a small amplitude trailing tail which will be of the same magnitude as that of the error of the approximation. Thus taking a finer grid or increasing the degree of the finite elements the trailing tails will be decreased in magnitude.

In Figure 6 we present a cross-section along the $x$-axis of the three solutions shown in Figure 5 at $T=25$. In this figure we can observe clearly the spurious oscillations generated due to the 


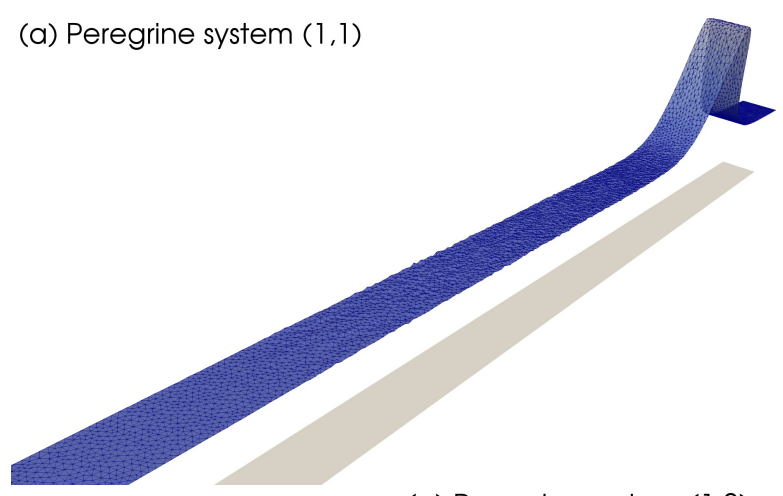

(b) BBM-BBM system $(1,1)$

(c) Peregrine system $(1,2)$
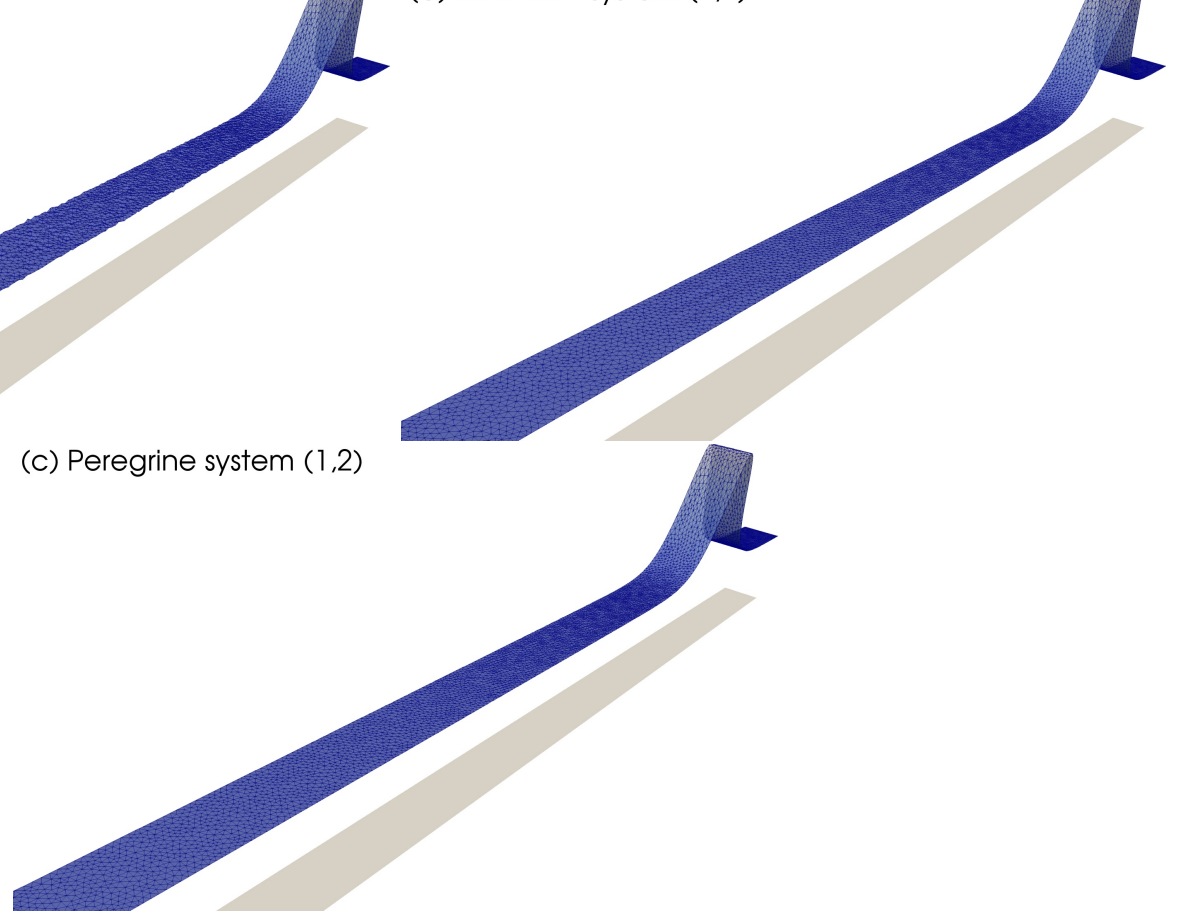

Figure 5. Propagation of a solitary wave of amplitude $A=0.3$ up to $T=25$. $(D=1, g=1)$

hyperbolic nature of the mass conservation equation of the Peregrine system with $P_{1}-P_{1}$ elements, which are significantly larger than the oscillations generated for the same system using $P_{1}-P_{2}$ elements or in the case of the BBM-BBM system with $P_{1}-P_{1}$ elements. In the same figure also we observe that in all cases the solitary wave travelled without significant change in shape and speed. The good behaviour of the finite element method with linear elements for similar BBMBBM systems has been observed also in $[24,25]$. A more interesting observation though here is that the trailing spurious oscillations are eliminated by the use of high-order Lagrange elements. For example, using quadratic elements for the discretization of the velocity field yields a clean solution presented in Figure $5(\mathrm{c})$. It is noted than in these experiments we considered $\Delta t=0.1$ and the same grid with the one used to generate the solitary waves with the Petviashvili method, Appendix A. The previous study of the convergence rates along with the present study of the propagation of the solitary waves suggest that the use of at least quadratic elements for the velocity field of the Peregrine system is mandatory for more accurate and highly-resolved numerical solutions of the Peregrine system. Alternatively, the use of the new regularized BBM-BBM system can be beneficial. In all the following numerical experiments we use linear elements in the approximation of the free-surface elevation and quadratic elements for the approximation of the velocity field.

\section{NuMERICAL EXPERIMENTS}

In this section we consider two different experimental settings that demonstrate the ability of the numerical method to handle the propagation and evolution of solitary waves in general twodimensional domains and variable bottom bathymetric features. Because there are not known exact formulas for the solitary wave solutions propagating without change in shape over a flat bottom $H_{0}$ of the systems under consideration, we considered the approximations of the solitary wave obtained using the Petviashvili method, see Appendix A. 

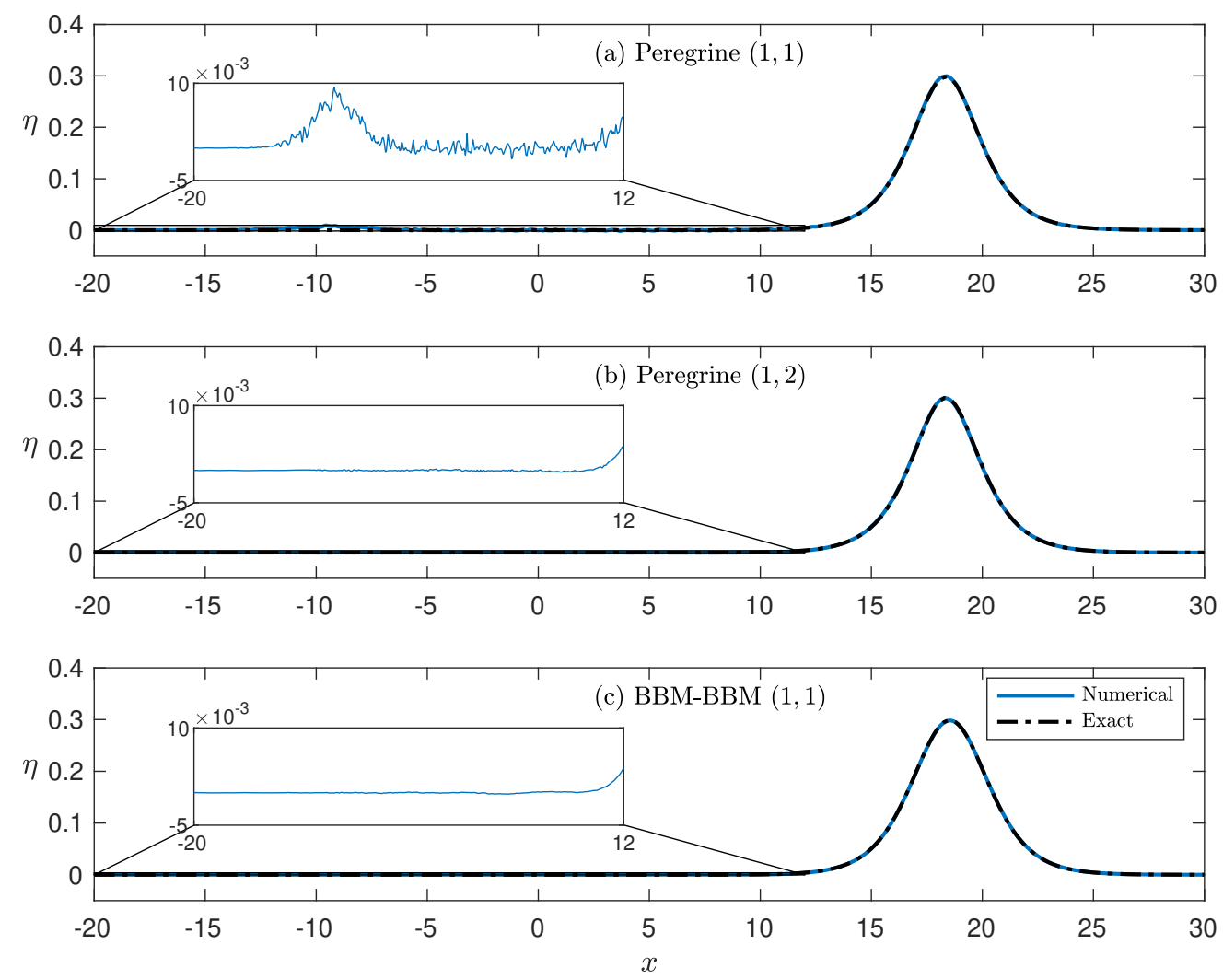

Figure 6. Cross-section along $x$-axis and comparison between the numerical and the exact solution at $T=25$

The experiments we use here, have been used before to validate similar mathematical and numerical models and seem to be appropriate for the regime of weakly nonlinear and weakly dispersive water waves. It is noted that in all the experiments we used $V_{h}^{1}$ for the finite element approximation of $\eta$ and $U_{h}^{2}$ for $\mathbf{u}$.

5.1. Scattering of solitary wave by a vertical cylinder. In this first experiment, the propagation of a small amplitude solitary wave and its interaction with a vertical cylinder is numerically investigated. The laboratory experiment was designed and presented in [10]. The particular experiment has been used for validation purposes for various Boussinesq systems [30]. In this experiment, a rectangular channel of dimensions $[-4,20] \times[0,0.55]$ was used. A vertical cylinder of diameter $0.16 \mathrm{~m}$ was placed at $(4.5,0.275)$. The solitary wave considered had amplitude $A=0.0375 \mathrm{~m}$. For the purposes of the numerical experiment we considered the same domain and the initial condition was translated such as its maximum pick was at $2.085 \mathrm{~m}$. The free-surface elevation $\eta$ was recorded in six wave-gauges located at wg1 $=(4.4,0.275)$, wg2 $=(4.5,0170)$, $\mathrm{wg} 3=(4.5,0.045), \mathrm{wg} 4=(4.6,0.275), \mathrm{wg} 5=(4.975,0.275), \mathrm{wg} 6=(5.375,0.275)$. The computational domain was discretised using a regular triangulation consisted of 22, 285 triangles. The timestep is taken $\Delta t=10^{-3}$. The triangulation and locations of the wave-gauges around the cylinder are presented in Figure 7.

In every case of Boussinesq system, the solitary wave propagates without significant change in shape (as there are no analytical solutions known for any of the systems it is expected that the approximate solitary wave will propagate as a solitary wave after shedding a small amplitude trailing dispersive tail). The recorded surface elevation at the wave-gauges agree with the experimental data 


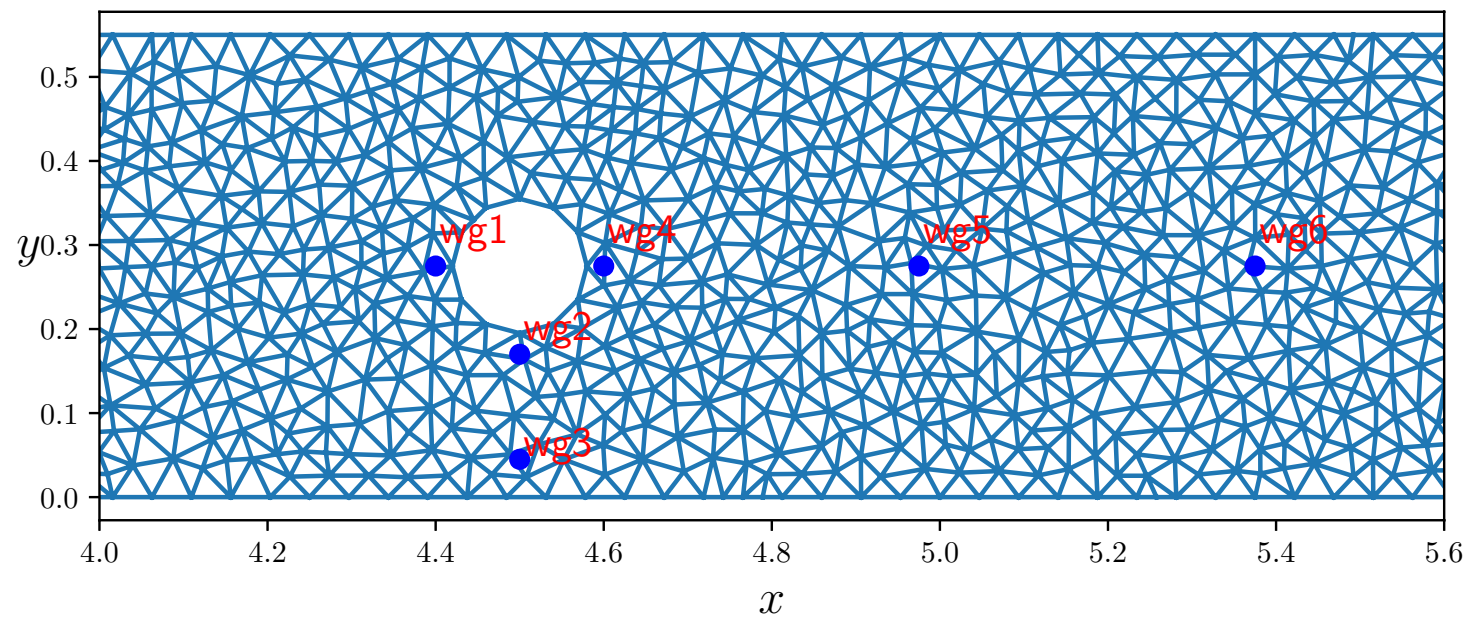

FiguRE 7. Triangulation around the cylinder and location of the wavegauges
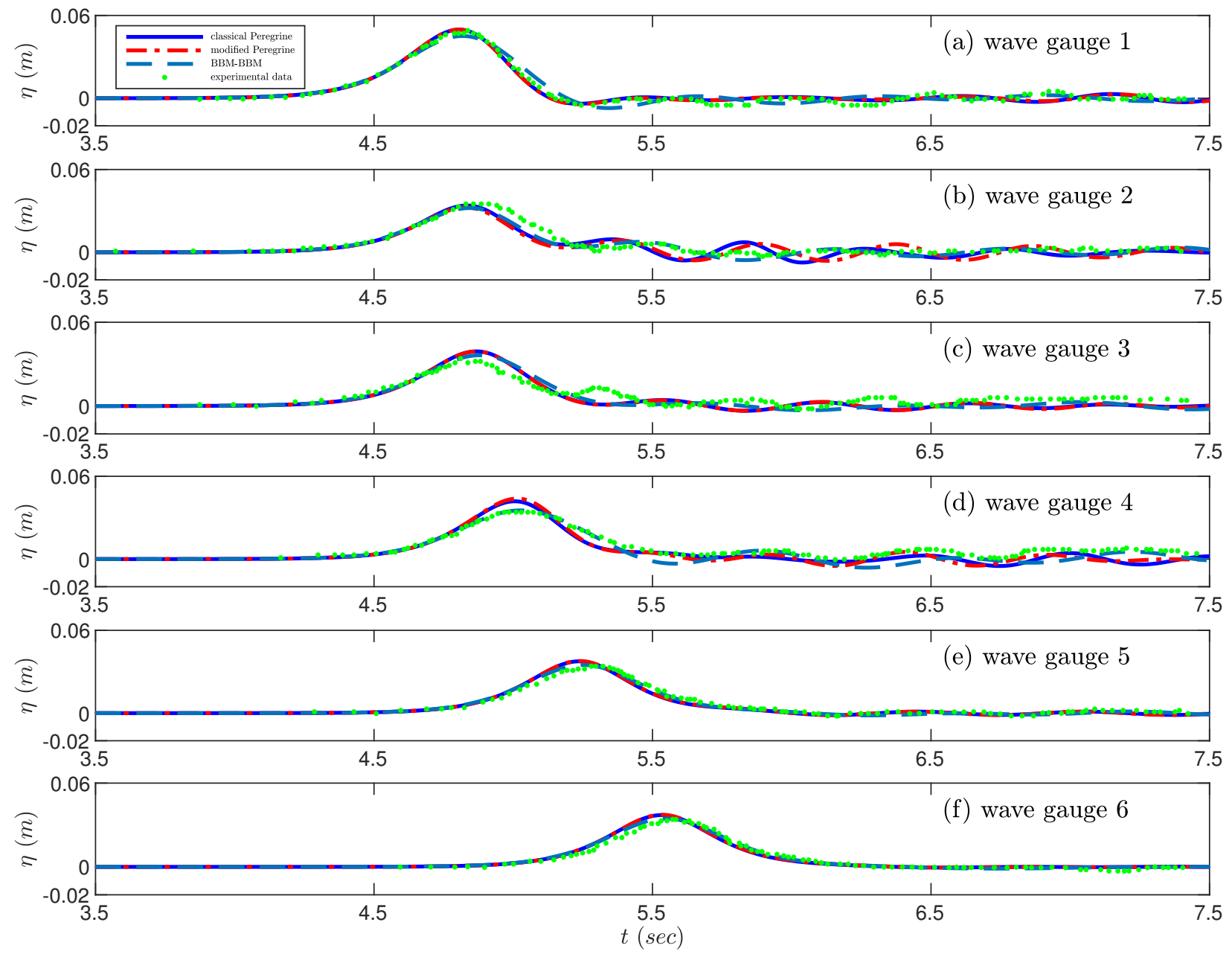

FiguRE 8. Recorded solution at the six wave-gauges 

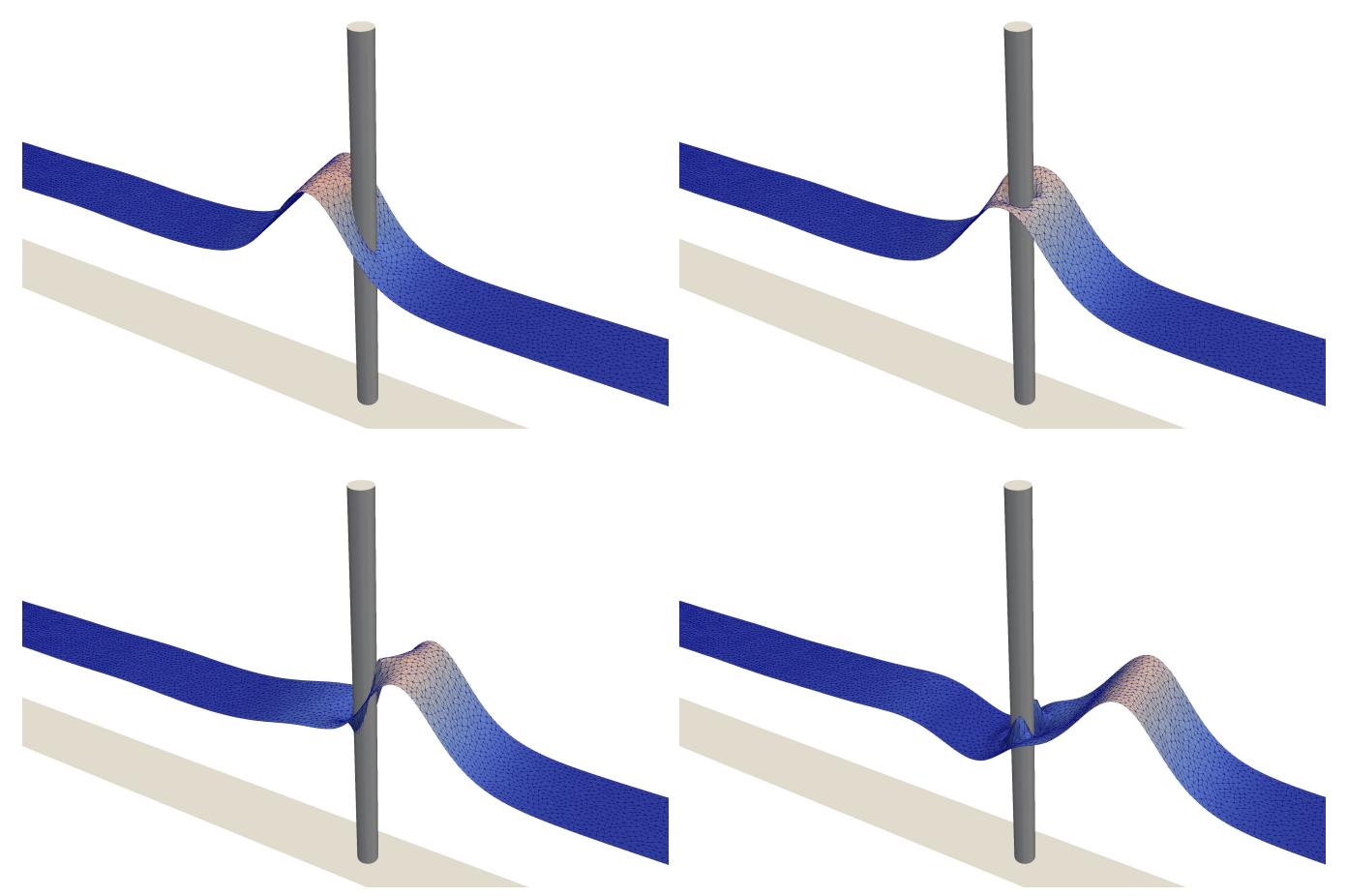

Figure 9. Interaction of the solitary wave with the cylinder at $t=4.688, t=$ $4.928, t=5.152, t=5.472$ (left to right, top to bottom), BBM-BBM system

of [10]. The results are presented in Figure 8. Apparently, the BBM-BBM system leads to very accurate results indicating that not only we achieved better convergence of the numerical method but also as a model is a good approximation of the Euler equations for the specific water wave regime. Compared to the numerical results of [10] we observe that the results obtained with the current finite element method using triangular grid are better. Possible explanation could be the better resolution of the computational domain and the higher-convergence properties because of the mixed finite element spaces we used. Compared to the results obtained using the optimised Nwogu's system in [30] again we observe that in both cases the results are very similar indicating that for the specific experiment, because the solitary wave has relatively small amplitude, the dispersion relation of the weakly nonlinear structure of the mathematical problems is not significant.

The interaction of the solitary wave with the cylinder causes the scattering of small amplitude dispersive waves. Due to the small width of the channel, the reflected waves propagate like one dimensional waves. On the other hand, as it was observed in similar numerical experiments with wide channel, these waves propagate as expanding waves in all directions, [26]. These features can be seen in a series of snapshots of the free surface $\eta(\mathbf{x}, t)$ in Figure 9 at various time instances, and with more details in Figure 10, where we present the results obtained with the BBM-BBM system.

5.2. Reflection of shoaling waves. In the second experiment we consider the shoaling of two solitary waves and their reflection by a vertical wall so as to study the efficiency of the numerical method in experiments with variable bathymetry. The specific experiments have been used before for the validation of various Boussinesq systems [22, 44, 35]. The propagation of the solitary waves is considered in a channel with dimensions $[-50,20] \times[0,1]$. The bottom topography in this experiment consists of a flat bottom of depth $0.7 m$ for $x<0$ and for all $y$ and a linear bathymetry 

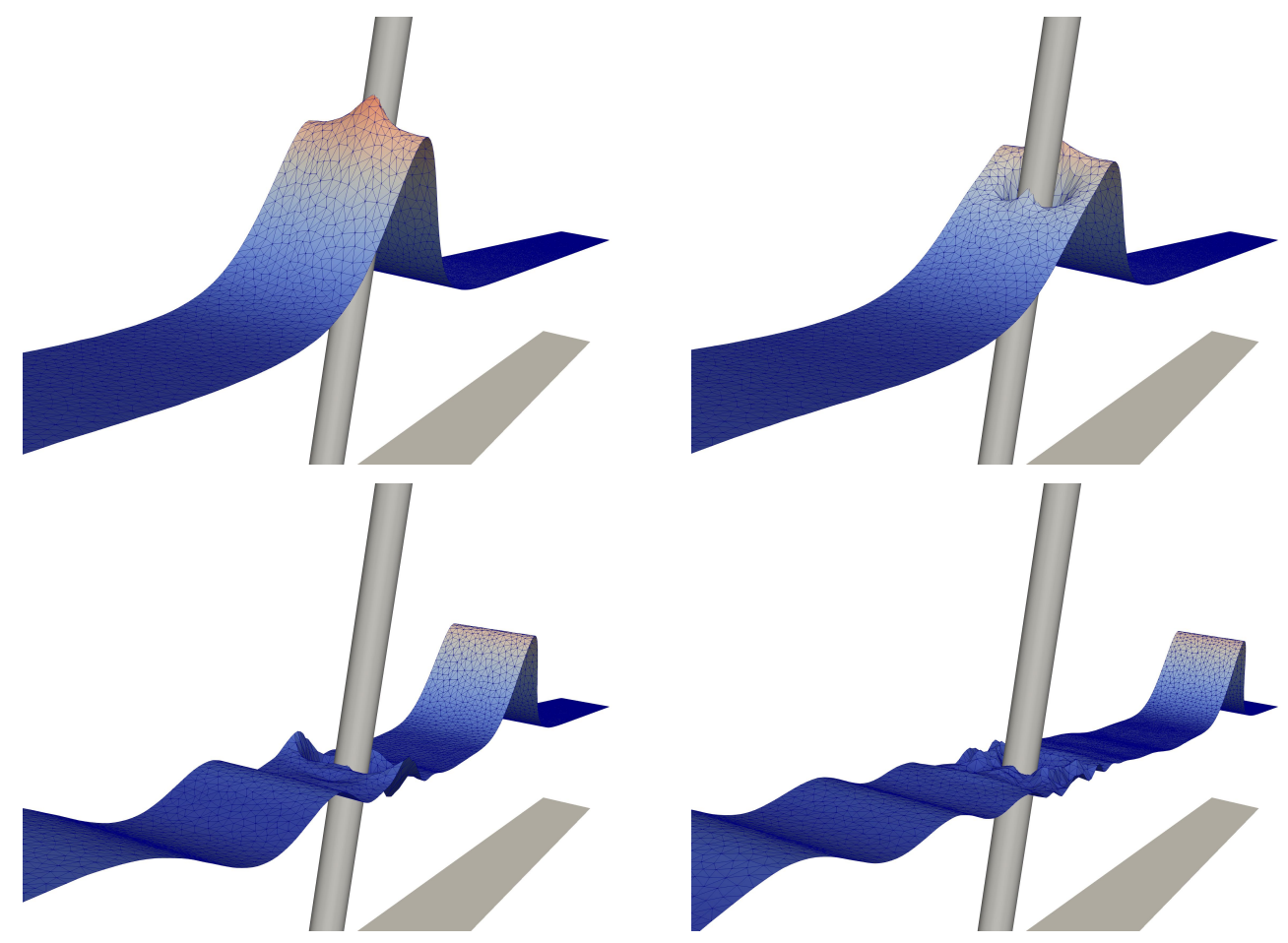

FiguRE 10. Interaction of the solitary wave with the cylinder at $t=4.832, t=$ $5.024, t=6.4, t=7.536$ (left to right, top to bottom), BBM-BBM system

of slope $1 / 50$ for $x \in[0,20]$ and for all $y$. The free-surface elevation is recorded by three wavegauges located at $(0.0,0.5),(16.25,0.5)$ and $(17.75,0.5)$. In the first case, the solitary wave has initial amplitude $0.07 \mathrm{~m}$. The computational domain was discretised using 14, 402 triangles. The time-step was taken $\Delta t=10^{-3}$.

Because of the small amplitude of the specific solitary wave and the relatively large depth, the solitary wave propagated without significant change of shape in all cases and the results fit very well with the laboratory data. Similar observations where made for other Boussinesq systems as well $[44,35]$ as the specific regime is in favour of all Boussinesq models. It is remarkable that the BBM-BBM system is again closed to the experimental data. The numerical method seems to be efficient during the shoaling and the reflection of the solitary wave. Figure 11 presents the recorded surface elevation at the three wave-gauges.

We also performed the same experiment with a solitary wave of amplitude $0.12 \mathrm{~m}$ over the same bottom as before. The numerical results obtained at the three wave-gauges are presented in Figure 12. Again the results are very satisfactory especially for the new BBM-BBM system as it appears, even in this case, the results are slightly better compared to the results obtained using the Peregrine system.

For the specific experiment the best performance has been observed by the Serre system which is appropriate for experiments with large amplitude solitary wave as there is no small-amplitude assumption in its derivation [35, 29].

5.3. Periodic waves over a submerged bar. In this section we test the numerical method against a laboratory experiment [11] designed to test the nonlinear and dispersive properties of Boussinesq systems with variable bathymetry. The specific experiment has been used in several works and is one of the standard benchmarks for numerical models $[21,30,44]$. In this experiment, 

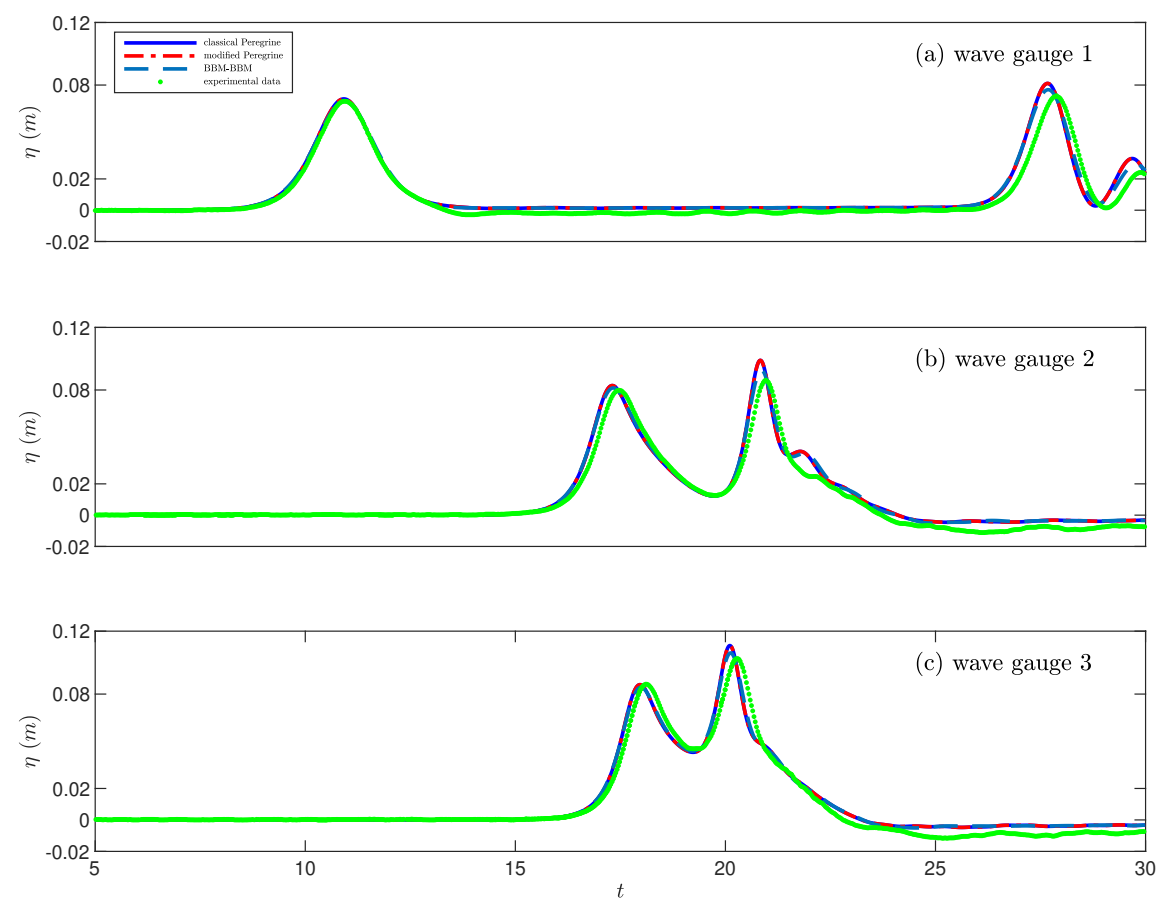

Figure 11. Surface elevation recorded at the three wave-gauges: Case $A=0.07$
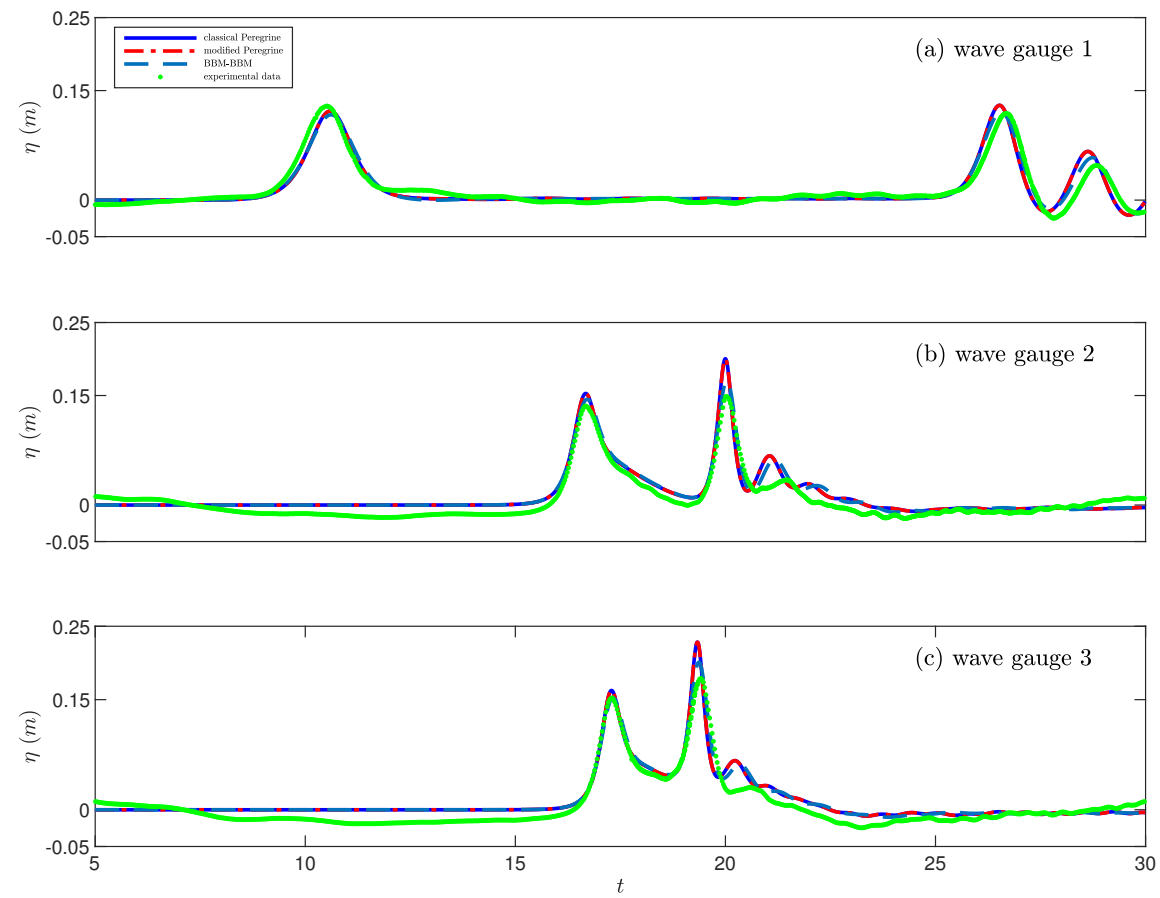

FiguRE 12. Surface elevation recorded at the three wave-gauges: Case $A=0.12$

small-amplitude periodic waves are generated by a wave-maker and propagate over a bathymetry 
defined by the function

$$
D(x, y)=\left\{\begin{array}{cc}
-0.05 x+0.7, & x \in[6,12) \\
0.1, & x \in[12,14) \\
0.1 x-1.3, & x \in[14,17] \\
0.4, & \text { elsewhere }
\end{array}\right.
$$

and depicted in Figure 13. When the generated waves interact with the upward part of the submerged bar, they shoal and become steep, generating higher-harmonics. Then the waves as they propagate over the downward part of the bar propagate faster covering a wide range of wavenmbers in the spectrum of long waves. In order to simulate the wave-maker we use the technique suggested in [44] by considering the time-dependent bottom topography incorporated into the Boussinesq systems (24) and (25). Specifically, we consider a time-dependent Gaussian bump centered at $x=2.01$ described by the function $\zeta(x, y, t)=a e^{-4(x-2.01)^{2}} \cos (-\omega t)$ with $\omega=2 \pi / \tau, \tau=2.02$ and $a=0.017$. The parameters of the moving part of the bottom have been chosen in accordance to [44]. As the bottom oscillates the periodic waves are generated at the free surface with period $k=2.02$. Although the experiment is typically one-dimensional, here we consider the rectangular domain $[-15,35] \times[0,1]$ and a triangulation consisted of 2,500 triangles. The timestep in this experiment is taken $\Delta t=0.1$. In order to avoid reflection from the lateral boundaries we also apply two sponge layers in the regions with $x \in[-15,0]$ and $x \in[25,35]$ represented by yellow color in Figure 13. The sponge layers are applied in each region by adding terms of the form $s(x) \cdot \eta$ in the mass conservation equation and using appropriate function $s(x)=c \exp \left(-a\left(x-x_{0}\right)^{2}\right)$. It is noted that the sponge layer terms do not require additional boundary conditions and that the amount of the reflected waves is of negligible importance. Similar boundary layers have been used in $[44,45]$. The free surface of the water is recorded at wave-gauges located at $x=10.5,12.5,13.5$, 14.5, 15.7 and 17.3. The locations of the wave-maker and the wave-gauges are depicted by vertical broken lines in Figure 13. The comparison of the free-surface elevation as predicted by the models

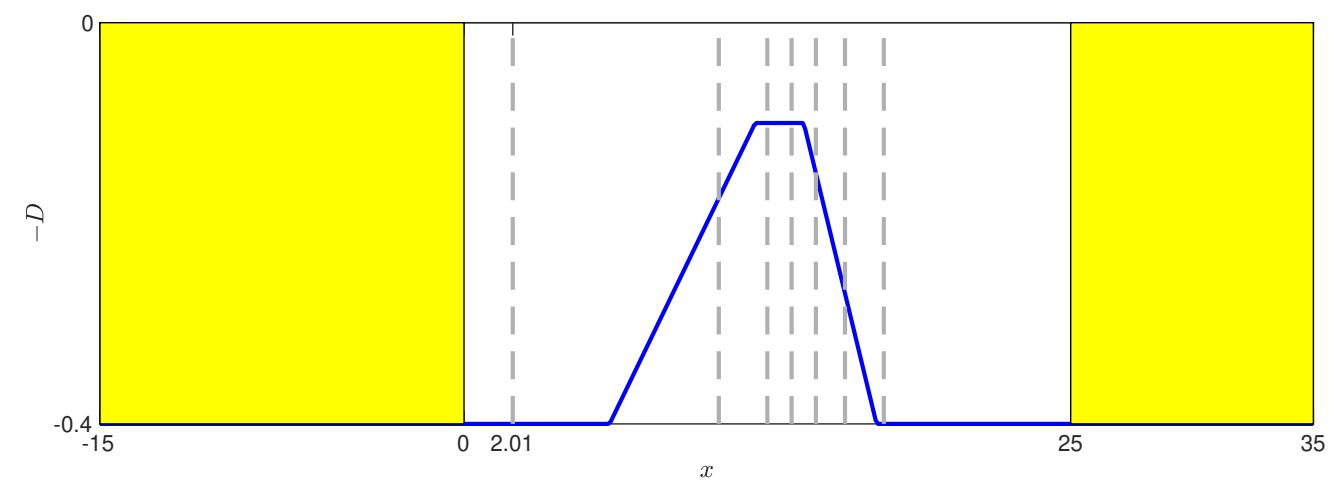

FIgURE 13. The bathymetry in relation to the sponge layers, wave maker and wave gauges locations

and the experimental data is shown in Figure 14. It is known that Boussinesq systems usually fail to predict the correct waves at the wave gauges located further away from the wave-maker as the waves generated are nearly breaking and thus outside of the long and small amplitude water wave regime. Boussinesq systems with improved dispersion and nonlinear characteristics, such as Nwogu's system [36], appear to have better behaviour in this experiment although again the errors can be large. In our case, both models have almost the same behaviour. As it was also observed in [11] for the Peregrine system, both numerical models start to diverge from the laboratory data after the wave-gauge $(\mathrm{d})$. Moreover, we observe that the numerical results obtained using the 

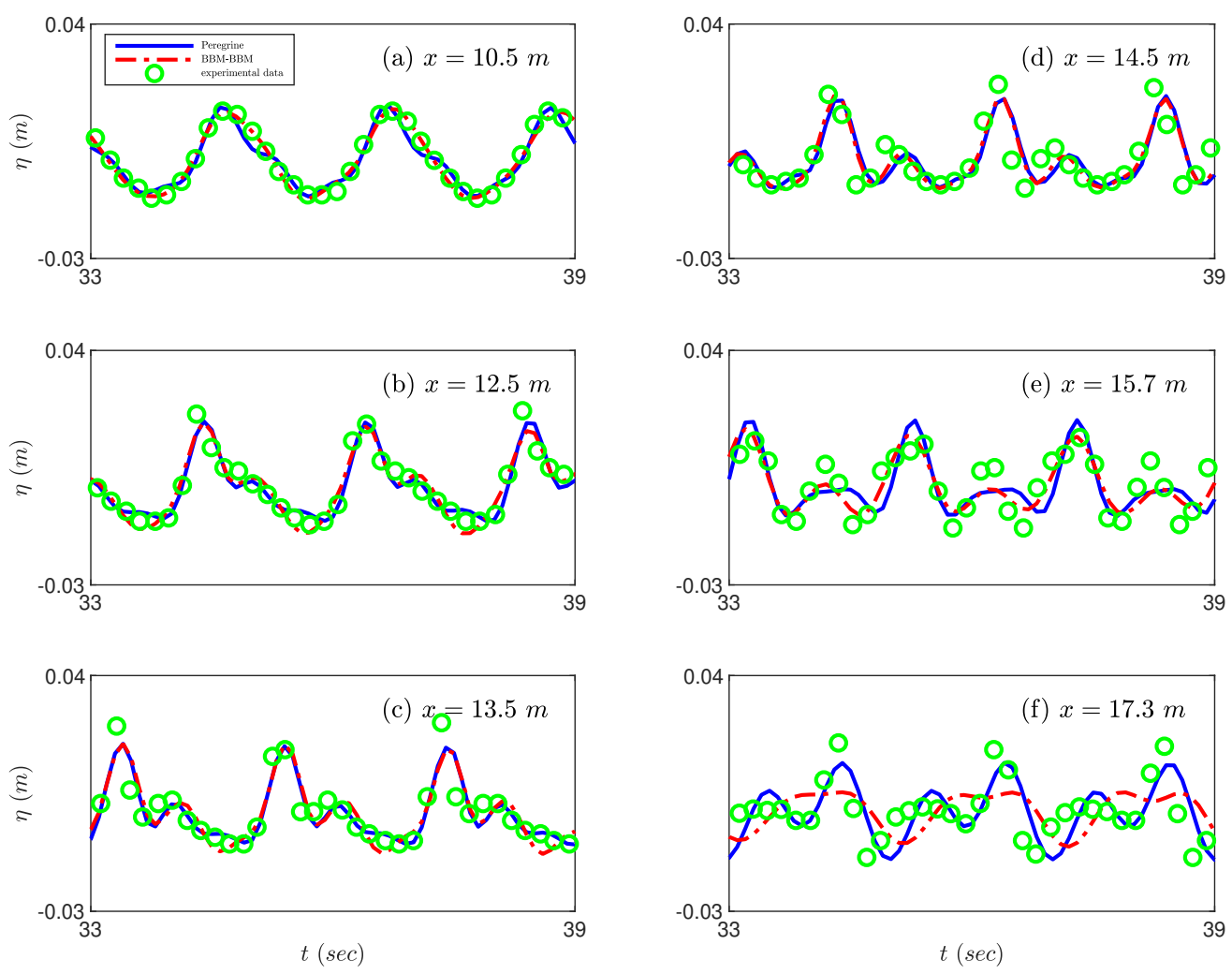

FigURE 14. Free-surface elevation recorded at six wave-gauges above the submerged mount

BBM-BBM system are in agreement with the numerical results obtained with Peregrine's system until the wave-gauge (e) located at $x=15.7$. After that, the two models start to diverge mainly because they have different dispersion relation from the Euler equations.

Remark 5.1. The results of the BBM-BBM system can approximate those obtained using Peregrine's system dramatically by considering for example $\theta=\sqrt{1 / 3}-0.01$, which on one hand has linear dispersion relation very close to the Peregrine system and in addition contains the regularization operator in the mass conservation equation. The numerical solution of the particular system is not presented here since it is very similar to the analogous solution of the Peregrine system.

Remark 5.2. In all the experiments, the mass conservation error was below $10^{-5}$.

\section{Conclusions And Perspectives}

The application of the standard Galerkin finite element method was studied in the case of the Peregrine system with slip-wall boundary conditions. A detailed study of the convergence rates revealed suboptimal convergence properties of the Galerkin method for the particular initialboundary value problem. Specifically, when we considered the same finite element space for all the dependent variables of the system, then the numerical solution $\eta_{h}$ converged to the free-surface elevation $\eta$ in the $L^{2}$-norm with rate of the order $O\left(h^{r+1 / 2}\right)$ for elements of degree $r$ with $r$ odd, and $O\left(h^{r}\right)$ when $r$ was even (when the optimal rate is $O\left(h^{r+1}\right)$ ). The convergence rate of the numerical solution $\mathbf{u}_{h}$ to the velocity field $\mathbf{u}$ was of order $O\left(h^{r}\right)$ independently of the degree $r$ except the case of linear element $r=1$ where the convergence was optimal. The convergence of both variables $\eta_{h}$ and $\mathbf{u}_{h}$ in $H^{1}$-norm is one degree smaller than the corresponding rate in $L^{2}$ norm. Surprisingly the convergence of $\mathbf{u}_{h}$ to $\mathbf{u}$ was optimal in the $\mathrm{H}(\mathrm{div})$-norm. Mixed finite 
element methods improved the convergence rates in $\eta$ but not in $\mathbf{u}$ which remained suboptimal. The suboptimal convergence rates in $\mathbf{u}$ are due to the significance of the regularization operator which is incomplete-elliptic. This conclusion was drawn after studying alternative systems with different nonlinearity (modified Peregrine system) and by replacing the incomplete-elliptic operator by the standard Laplacian (simplified Peregrine system) using asymptotic arguments. Although the simplified Peregrine system has better convergence properties, it is hard to be used in situations with slip-wall boundary conditions as it requires the velocity field to be zero on the boundary.

Another problematic situation occurs in the Galerkin method for the Peregrine system when piecewise linear elements are used in two-space dimensions, namely, spurious oscillations can appear behind smooth non-breaking waves propagating over flat bottom. This phenomenon appears because of the hyperbolic structure of the mass conservation equation of the Peregrine system, and can be resolved by using piecewise quadratic (or other high-order) Lagrange elements for the velocity field and/or the free-surface elevation.

A new Boussinesq system of BBM-BBM type was also proposed as an alternative to Peregrine's system. The momentum conservation equation of the new system has the same structure with the respective equation of the Peregrine system while the mass conservation equation has the classical elliptic regularization operator which regularizes the solution so that no spurious oscillations can be observed even with the use of piecewise linear elements. Moreover, the convergence rates for the free-surface elevation variable are optimal, while the convergence rates for the velocity field is optimal again only in the $\mathrm{H}(\mathrm{div})$-norm following the same convergence pattern as the Peregrine system. This phenomenon is due to the variable bottom topography and the Nitsche method used to approximate the slip-wall boundary conditions. In the case of flat topography the convergence rate for the velocity field is optimal even in the $L^{2}$ norm.

All the numerical models were tested against standard benchmark experiments within the region of validity of the Boussinesq systems. In particular, we studied the scattering of a line solitary wave by a vertical cylinder, the shoaling of solitary waves and their reflection by a vertical wall and the interaction of periodic waves propagating over an underwater obstacle. The comparison between the new BBM-BBM and the Peregrine systems showed that both systems share the same accuracy against the experimental data for the specific experiments. Thus, the new system can be used in principle as a good alternative to the Peregrine system.

Finally, because there are not any known analytical formulas for solitary wave solutions in closed form of any of the aforementioned systems we employed the Petviashvili method for the computation of numerical approximations of the line solitary waves into the same finite element spaces used for the discretization of the partial differential equations. The convergence and the speed of the Petviashvili method was unexpectedly fast. Further studies for this particular numerical method will follow in the future.

The main conclusions and perspectives are summarised here:

- The Galerkin finite element method for Boussinesq systems including other Boussinesq type systems where the mass conservation has the form of a hyperbolic conservation law, requires the use of at least quadratic Lagrange elements in the approximation of the velocity field to achieve satisfactory resolution of the numerical solution.

- Despite the fact that BBM-BBM type systems have less accurate linear dispersion relationship compared to Nwogu's system, it seems that in practical problems related to small amplitude long waves, they appear to be as accurate as the Peregrine system.

- The Petviashvili method applied to the solution of nonlinear equations resulted from the discretization of partial differential equations with finite element methods appear to be very efficient and with good convergence properties. 


\section{ACKNowledGements}

DM would like to thank King Abdullah University of Science and Technology(KAUST) Saudi Arabia, for its warm hospitality and support during this project. DM was supported also by the Victoria University of Wellington RSL fund and the Marsden fund administered by the Royal Society of New Zealand. This work is dedicated to Prof. Vassilios Dougalis on the occasion of his $70^{\text {th }}$ birthday.

\section{Appendix A. The Petviashvili iteration}

In nonlinear and dispersive wave systems of Boussinesq type, although there is well-established theory for the existence of classical solitary wave solutions, there are only a few cases of analytical formulas for traveling wave solutions [16, 17, 23]. For the classical Boussinesq system the only formulas known for traveling wave solutions have $\eta=-D_{0}$. This means that there is no water, but a solitary wave profile in the velocity field. These traveling wave solutions, although are useful for testing purposes, they have no physical context. For BBM-BBM type systems one can find analytical formulas for traveling wave solutions which again are not of the $\mathrm{sech}^{2}$-form and their negative excitation is bellow the bottom topography $D_{0}[16,17]$. Since the exact formulas for traveling wave solutions known for these systems are not solitary waves of the classical sense [23], then if physically relevant solitary wave solutions are required one should employ numerical methods for their computation. In the sequel we describe a numerical method commonly used for such computations in the context of Galerkin / finite element method.

The Petviashvili method [40], is a modified fixed point method for solving nonlinear equations originally derived from equations for solitary waves. Although Petviashvili's method has been applied and studied analytically using Fourier / pseudospectral methods [38, 4] it has been applied successfully with Galerkin finite element methods as well [37].

Here we first present the Petviashvili iteration for the approximation of the line solitary waves of the Peregrine system using a Galerkin finite element method. Assume that we seek traveling wave solutions of the form

$$
\eta(\mathbf{x}, t)=\eta(\boldsymbol{\xi}), \quad \mathbf{u}(\mathbf{x}, t)=\mathbf{u}(\boldsymbol{\xi}),
$$

where $\boldsymbol{\xi}=(\xi, \zeta)$ with $\xi=\boldsymbol{\alpha} \mathbf{x}-c_{s} t-\mathbf{x}_{0}, \mathbf{x}_{0} \in \mathbb{R}$, and $\zeta=\boldsymbol{\alpha}^{\perp} \mathbf{x}$. This represents a traveling wave solution propagating with constant speed $c_{s}$, without change in shape, in a channel aligned along the direction of the vector $\boldsymbol{\alpha}=\left(\alpha_{x}, \alpha_{y}\right)^{T},|\boldsymbol{\alpha}|=1$ over a flat bottom $D(\mathbf{x})=D_{0}$, that tends to zero at infinity, while there are no restrictions along the vertical to $\boldsymbol{\alpha}$ direction $\boldsymbol{\alpha}^{\perp}=\left(\alpha_{y},-\alpha_{x}\right)^{T}$. Because the domain for the numerical simulations must be bounded, we assume that the channel is long enough so as the exponentially decreasing solitary wave fits along the $\boldsymbol{\alpha}$ direction in the sense that at the edges of the domain the solitary wave is practically zero. Substituting the ansatz (34) into Peregrine's equations (1) we obtain the system of partial differential equations

$$
\begin{aligned}
& -c_{s} \eta_{\xi}+\nabla_{\xi \zeta} \cdot\left(D_{0}+\eta\right) A \mathbf{u}=0 \\
& -c_{s} \mathbf{u}_{\xi}+g A \nabla_{\xi \zeta} \eta+\left(\mathbf{u} \cdot A \nabla_{\xi \zeta}\right) \mathbf{u}+c_{s} \frac{1}{3} D_{0}^{2} A \nabla_{\xi \zeta}\left(\nabla_{\xi \zeta} \cdot A \mathbf{u}_{\xi}\right)=0
\end{aligned}
$$

where $\nabla_{\xi \zeta}=\left(\partial_{\xi}, \partial_{\zeta}\right)^{T}$ and

$$
A=\left(\begin{array}{cc}
\alpha_{x} & \alpha_{y} \\
\alpha_{y} & -\alpha_{x}
\end{array}\right) .
$$

Multiplying the second equation in (35) with $A$ and denoting $\mathbf{w}=(w, \tilde{w})$ where

$$
w=\boldsymbol{\alpha} \cdot \mathbf{u}, \quad \tilde{w}=\boldsymbol{\alpha}^{\perp} \cdot \mathbf{u},
$$


then the system (35) becomes

$$
\begin{aligned}
& -c_{s} \eta_{\xi}+\nabla_{\xi \zeta} \cdot\left(D_{0}+\eta\right) \mathbf{w}=0 \\
& -c_{s} \mathbf{w}_{\xi}+g \nabla_{\xi \zeta} \eta+\left(\mathbf{w} \cdot \nabla_{\xi \zeta}\right) \mathbf{w}+c_{s} \frac{1}{3} D_{0}^{2} \nabla_{\xi \zeta}\left(\nabla_{\xi \zeta} \cdot \mathbf{w}_{\xi}\right)=0 .
\end{aligned}
$$

Assuming that the solution is constant along the direction of the vector $\boldsymbol{\alpha}^{\perp}$ then system (37) can be simplified after integration into the system

$$
\begin{aligned}
-c_{s} \eta+\left(D_{0}+\eta\right) w & =0, \\
-c_{s} w+g \eta+\frac{1}{2} w^{2}+c_{s} \frac{1}{3} D_{0}^{2} w_{\xi \xi} & =0, \\
\tilde{w} & =0,
\end{aligned}
$$

where we intentionally keep the dependence of the equations on $\tilde{w}$ to emphasize the fact that we are looking for two-dimensional waves and also for implementational purposes. Eliminating $\eta$ in (38) we obtain the relation

$$
\eta=\frac{D_{0} w}{c_{s}-w},
$$

between the unknowns $\eta$ and $w$ and the partial differential equation

$$
\mathcal{L} \mathbf{w}=\mathcal{N}(\mathbf{w}),
$$

with

$$
\mathcal{L}=\left(\begin{array}{cc}
c_{s}-\frac{1}{3} D_{0}^{2} c_{s} \partial_{\xi \xi} & 0 \\
0 & 1
\end{array}\right) \text { and } \mathcal{N}(\mathbf{w})=\left(\begin{array}{c}
\frac{1}{2} w^{2}+g \frac{D_{0} w}{c_{s}-w} \\
0
\end{array}\right)
$$

Solving the equation (40) for $w$ we recover the free-surface elevation from (39). In order to solve (40) we employ again the standard Galerkin finite element method: seek $\mathbf{w}_{h} \in U_{h}^{r_{2}}$ satisfying the equation written in the weak form

$$
\mathcal{L}_{h}\left(\mathbf{w}_{h}, \chi\right)=\left(\mathcal{N}\left(\mathbf{w}_{h}\right), \chi\right), \quad \text { for all } \quad \chi \in U_{h}^{r_{2}},
$$

where $\mathcal{L}_{h}(\mathbf{w}, \boldsymbol{\chi})$ is the bilinear form

$$
\mathcal{L}_{h}(\mathbf{w}, \boldsymbol{\chi})=c_{s}(w, \phi)+\frac{1}{3} D_{0}^{2} c_{s}\left(w_{\xi}, \phi_{\xi}\right)+(\tilde{w}, \psi),
$$

defined for all $\mathbf{w}=(w, \tilde{w}) \in U_{h}^{r_{2}}$ and $\chi=(\phi, \psi) \in U_{h}^{r_{2}}$. In order to solve the nonlinear equation (42), we use the Petrviashvilli's iteration defined as

$$
\mathcal{L}_{h}\left(\mathbf{w}_{h}^{n+1}, \boldsymbol{\chi}\right)=M_{n}^{\gamma}\left(\mathcal{N}\left(\mathbf{w}_{h}^{n}\right), \chi\right), \quad \forall \chi \in U_{h}^{r_{2}}, \quad n=0,1, \cdots,
$$

where $M_{n}$ is the scalar

$$
M_{n}=\frac{\mathcal{L}_{h}\left(\mathbf{w}_{h}^{n}, \mathbf{w}_{h}^{n}\right)}{\left(\mathcal{N}\left(\mathbf{w}_{h}^{n}\right), \mathbf{w}_{h}^{n}\right)} .
$$

The function $\mathbf{w}_{h}^{0}$ can be the $L^{2}$-projection of any initial guess $\mathbf{w}^{0}$ of the solitary wave solution onto the finite element space $V_{h}^{r_{1}}$. As an approximation to the line solitary waves of the Boussinesq systems we considered the functions

$$
\begin{aligned}
\eta^{0}(\boldsymbol{\xi}) & =A \operatorname{sech}^{2}(\lambda \xi), \\
w^{0}(\boldsymbol{\xi}) & =c_{s} \eta^{0}(\boldsymbol{\xi}) /\left(D_{0}+\eta^{0}(\boldsymbol{\xi})\right), \\
\tilde{w}^{0}(\boldsymbol{\xi}) & =0 .
\end{aligned}
$$


with $c_{s}=\sqrt{g\left(D_{0}+A\right)}$, and $\lambda=\sqrt{3 A / 4 D_{0}^{3}}$. The exponent $\gamma$ is taken to be equal to 2 but it can be any number in the interval $[1,3]$. Computing the approximation $w_{h}$, we can construct the function $\eta_{h}$ by considering the $L^{2}$-projections of (35) in $V_{h}^{r_{1}}$, while the velocity field $\mathbf{u}_{h}$ can be recovered by solving the linear system (36).

Substitution of the ansatz (34) into the BBM-BBM system (23) with flat bottom $D(\mathbf{x})=D_{0}$ and following similar computations as before, leads to the system

$$
\begin{aligned}
-c_{s} \eta+\left(D_{0}+\eta\right) w+\frac{1}{6} c_{s} D_{0}^{2} \nabla_{\xi \zeta}^{2} \eta & =0, \\
-c_{s} w+g \eta+\frac{1}{2} w^{2}+\frac{1}{6} c_{s} D_{0}^{2} w_{\xi \xi} & =0 \\
\tilde{w} & =0 .
\end{aligned}
$$

In this case we cannot eliminate the variable $\eta$ as in the Peregrine system so instead of solving a scalar ordinary differential equation we practically need to solve a system of equations that involves also the variable $\eta$. The system (46) can be written in the form

$$
\tilde{\mathcal{L}} \mathbf{w}=\tilde{\mathcal{N}}(\mathbf{w}),
$$

with $\mathbf{w}=(\eta, w, \tilde{w})^{T}$ and

$$
\tilde{\mathcal{L}}=\left(\begin{array}{ccc}
c_{s}-\frac{1}{6} c_{s} D_{0}^{2} \nabla_{\xi \zeta}^{2} & -D_{0} & 0 \\
-g & c_{s}-\frac{1}{6} c_{s} D_{0}^{2} \partial_{\xi}^{2} & 0 \\
0 & 0 & 1
\end{array}\right) \quad \text { and } \quad \tilde{\mathcal{N}}(\mathbf{w})=\left(\begin{array}{c}
\eta w \\
\frac{1}{2} w^{2} \\
0
\end{array}\right)
$$

The Galerkin finite element method for solving system (47)-(48) is then expressed as follows: week for the approximation $\mathbf{w}_{h}:=\left(\eta_{h}, w_{h}\right)^{T} \in V_{h}^{r_{1}} \times U_{h}^{r_{2}}$, such that

$$
\tilde{\mathcal{L}}_{h}\left(\mathbf{w}_{h}, \chi\right)=\left(\tilde{\mathcal{N}}\left(\mathbf{w}_{h}\right), \chi\right), \quad \text { for all } \quad \chi \in V_{h}^{r_{1}} \times U_{h}^{r_{2}},
$$

where $\tilde{\mathcal{L}}_{h}$ is the bilinear form

$$
\begin{aligned}
\tilde{\mathcal{L}}_{h}(\mathbf{w}, \boldsymbol{\chi}) & =c_{s}(\eta, \phi)+\frac{1}{6} c_{s} D_{0}^{2}\left(\nabla_{\xi \zeta} \eta, \nabla_{\xi \zeta} \phi\right)-D_{0}(w, \phi) \\
& +c_{s}(w, \chi)+\frac{1}{6} c_{s} D_{0}^{2}\left(w_{\xi}, \chi_{\xi}\right)-g(\eta, \chi)+(\tilde{w}, \psi),
\end{aligned}
$$

for all $\mathbf{w}=(\eta, w, \tilde{w})^{T} \in V_{h}^{r_{1}} \times U_{h}^{r_{2}}$ and $\chi=(\phi, \chi, \psi)^{T} \in V_{h}^{r_{1}} \times U_{h}^{r_{2}}$. Given an initial guess for $\mathbf{w}_{h}^{0}$ for the solitary wave solution of the BBM-BBM system, the Petviashvili method for solving the nonlinear system of equations (49) is then defined similarly to the analogous method for the Peregrine system:

$$
\tilde{\mathcal{L}}_{h}\left(\mathbf{w}_{h}^{n+1}, \boldsymbol{\chi}\right)=\tilde{M}_{n}^{\gamma}\left(\tilde{\mathcal{N}}\left(\mathbf{w}_{h}^{n}\right), \boldsymbol{\chi}\right), \quad n=0,1, \cdots,
$$

for all $\chi \in V_{h}^{r_{1}} \times U_{h}^{r_{2}}$ and $\tilde{M}_{n}$ the scalar

$$
\tilde{M}_{n}=\frac{\tilde{\mathcal{L}}_{h}\left(\mathbf{w}_{h}^{n}, \mathbf{w}_{h}^{n}\right)}{\left(\tilde{\mathcal{N}}\left(\mathbf{w}_{h}^{n}\right), \mathbf{w}_{h}^{n}\right)} .
$$

The initial guess for the solitary wave solution can be taken again to be (45).

As a stopping criterion for the Petviashvili's iteration we consider the norm of the normalized residual to be less than a prescribed tolerance $\delta$. Specifically, we consider the stopping criderion

$$
R_{n}=\left|\tilde{\mathcal{L}}_{h}\left(\mathbf{w}_{h}^{n}, \mathbf{w}_{h}^{n}\right)-\left(\tilde{\mathcal{N}}\left(\mathbf{w}_{h}^{n}\right), \mathbf{w}_{h}^{n}\right)\right| /\left\|\mathbf{w}_{h}^{n}\right\|_{2}<\delta,
$$

with $\delta$ appropriate tolerance. In this paper we take $\delta=10^{-5}$. In all the experiments we tested the convergence tolerance was satisfied in less than 20 iterations. For example, when we consider the horizontal channel $[-20,30] \times[-1,1]$ and we seek for solitary waves traveling in the direction 
$\boldsymbol{\alpha}=(1,0)^{T}$ with depth $D_{0}=1$ and $g=1$ the Petviashvili method converged to a solitary wave of amplitude $A=0.3$ with tolerance $\delta=10^{-5}$ in 5 iterations for the BBM-BBM system and in 16 iterations for the Peregrine system. For this experiment we considered an unstructured grid of 9, 510 triangles with maximum diameter 0.24 and minimum 0.09 . The propagation of this solitary wave is presented in Section 4.1.

Remark A.1. The exact relationship between speed and amplitude is known only for the Peregrine system [6], which is

$$
c_{s}=\frac{\sqrt{6}\left(D_{0}+A\right)}{\sqrt{3 D_{0}+2 A}} \frac{\sqrt{g D_{0}\left(D_{0}+A\right) \log \left(\frac{D_{0}+A}{D_{0}}\right)-g D_{0} A}}{A} .
$$

For the BBM-BBM system, where there is not any known speed-amplitude relationship, the appropriate solitary wave can be achieved using a continuation method.

\section{REFERENCES}

[1] K. Adamy. Existence of solutions for a boussinesq system on the half line and on a finite interval. Discrete Continuous Dynamical Syst. A, 29:25-49, 2011.

[2] A. Ali and H. Kalisch. Mechanical balance laws for Boussinesq models of surface water waves. J. Nonlinear Sci., 22:371-398, 2012.

[3] M. Alnaes, J. Blechta, J. Hake, A. Johansson, B. Kehlet, A. Logg, C. Richardson, J. Ring, M. Rognes, and G. Wells. The FEniCS Project Version 1.5. Archive of Numerical Software, 3(100):9-23, 2015.

[4] J. Álvarez and A. Durán. Petviashvili type methods for traveling wave computations: I. Analysis of convergence. J. Comp. Appl. Math., 266:39-51, 2014.

[5] D. Ambrosi and L Quartapelle. A Taylor-Galerkin method for simulating nonlinear dispersive water waves. J. Comp. Phys., 146:546-569, 1998.

[6] D. Antonopoulos and V. Dougalis. Numerical solution of the "classical" Boussinesq system. Math. Comp. Simul., 82:984-1007, 2012.

[7] D. Antonopoulos and V. Dougalis. Error estimates for Galerkin approximations of the "classical" Boussinesq system. Math. Comp., 82:689-717, 2013.

[8] D. Antonopoulos and V. Dougalis. Notes on error estimates for Galerkin approximations of the "classical" Boussinesq system and related hyperbolic problems. arXiv preprint arXiv:1008.4248, University of Athens, Greece, 2013.

[9] D. Antonopoulos, V. Dougalis, and D. Mitsotakis. Numerical solution of Boussinesq systems of the Bona-Smith family. Appl. Num. Math., 60:314-336, 2010.

[10] J.S. Antunes do Carmo, F.J. Seabra-Santos, and Barthélemy E. Surface waves propagation in shallow water: a finite element model. Int. J. Numer. Meth. Fluids, 16:447-459, 1993.

[11] S Beji and JA Battjes. Numerical simulation of nonlinear wave propagation over a bar. Coastal Engineering, 23:1-16, 1994.

[12] T. B Benjamin, J. L Bona, and J. J Mahony. Model equations for long waves in nonlinear dispersive systems. Philosophical Transactions of the Royal Society of London. Series A, Mathematical and Physical Sciences, 272(1220):47-78, 1972 .

[13] J. Bona, M. Chen, and J.-C. Saut. Boussinesq equations and other systems for small-amplitude long waves in nonlinear dispersive media. i: Derivation and linear theory. J.Nonlin. Sci., 12(4), 2002.

[14] J. Bona, T. Colin, and D. Lannes. Long wave approximations for water waves. Arch. Rat. Mech. Anal., 178:373410, 2005.

[15] J. Bona and R. Smith. A model for the two-way propagation of water waves in a channel. Math. Proc. Camb. Phil. Soc., 79:167-182, 1976.

[16] M. Chen. Exact traveling-wave solutions to bidirectional wave equations. International Journal of Theoretical Physics, 37:1547-1567, 1998.

[17] M. Chen. Solitary-wave and multi-pulsed traveling-wave solutions of boussinesq systems. Applicable Analysis, $75: 213-240,2000$.

[18] M. Chen. Equations for bi-directional waves over an uneven bottom. Math. Comp. Simul., 62:3-9, 2003.

[19] P. G. Ciarlet. The finite element method for elliptic problems, volume 40. SIAM Philadelphia, 2002.

[20] L. Debnath. Nonlinear partial differential equations for scientists and engineers. Birkhäuser Bassel, 2011. 
[21] M.W. Dingemans. Comparison of computations with Boussinesq-like models and laboratory measurements. Technical Report H1684.12, MAST G8M, Coastal Morphodynamics Research Programme, 1994.

[22] N. Dodd. Numerical model of wave run-up, overtopping, and regeneration. J. Waterway Port Coastal and Oc Eng, 124(2):73-81, 1998.

[23] V. Dougalis and D. Mitsotakis. Theory and numerical analysis of boussinesq systems: A review. In N. Kampanis, V. Dougalis, and J. Ekaterinaris, editors, Effective Computational Methods in Wave Propagation, pages 63-110. CRC Press, 2008.

[24] V. Dougalis, D. Mitsotakis, and J.-C. Saut. On some Boussinesq systems in two space dimensions: Theory and numerical analysis. ESAIM: Math. Model. Num. Anal., 41:825-854, 2007.

[25] V. Dougalis, D. Mitsotakis, and J.-C. Saut. On initial-boundary value problems for a Boussinesq system of BBM-BBM type in a plane domain. Discrete Contin. Dyn. Syst, 23:1191-1204, 2009.

[26] V. Dougalis, D. Mitsotakis, and J.-C. Saut. Boussinesq systems of Bona-Smith type on plane domains: theory and numerical analysis. J. Sci. Comp., 44(2):109-135, 2010.

[27] V. Duchêne and S. Israwi. Well-posedness of the Green-Naghdi and Boussinesq-Peregrine systems. to appear in Ann. Math. Blaise Pasca, 2018.

[28] S.D. Hatland and H. Kalisch. Wave breaking in undular bores generated by a moving weir. Physics of Fluids, 31(3):033601, 2019.

[29] H. Kalisch, Z. Khorsand, and D. Mitsotakis. Mechanical balance laws for fully nonlinear and weakly dispersive water waves. Phys. D, 333:243-253, 2016.

[30] M. Kazolea, A. Delis, I. Nikolos, and C. Synolakis. An unstructured finite volume numerical scheme for extended 2D Boussinesq-type equations. Coastal Engineering, 69:42-66, 2012.

[31] G. S. Khakimzyanov and D. Dutykh. Long wave interaction with a partially immersed body. Part I: Mathematical models. Comm. Comp. Phys., pages 1-62, 2019.

[32] Y.S. Li, S.-X. Liu, Y.-X. Yu, and G.-Z. Lai. Numerical modeling of Boussinesq equations by finite element method. Coastal engineering, 37:97-122, 1999.

[33] A. Logg, K.-A. Mardal, and G. Wells. Automated Solution of Differential Equations by the Finite Element Method. Number 978-3-642-23098-1 in Lecture Notes in Computational Science and Engineering. Springer-Verlag Berlin Heidelberg, 2012.

[34] D. Mitsotakis. Boussinesq systems in two space dimensions over a variable bottom for the generation and propagation of tsunami waves. Mat. Comp. Simul., 80:860-873, 2009.

[35] D. Mitsotakis, S. Synolakis, and M. McGuinness. A modified galerkin/finite element method for the numerical solution of the serre-green-naghdi system. Int. J. Num. Meth. Fluids, 83:755-778, 2017.

[36] O. Nwogu. Alternative form of boussinesq equations for nearshore wave propagation. J. Waterway Port Coastal and Oc Eng, 119(6):618-638, 1993.

[37] D. Olson, S. Shukla, G. Simpson, and D. Spirn. Petviashvilli's method for the Dirichlet problem. J. Sci. Comp., 66(1):296-320, 2016.

[38] D. Pelinovsky and Y. Stepanyants. Convergence of Petviashvili's iteration method for numerical approximation of stationary solutions of nonlinear wave equations. SIAM J. Nume. Anal., 42(3):1110-1127, 2004.

[39] D. Peregrine. Long waves on a beach. J. Fluid Mech., 27:815-827, 1967.

[40] V.I. Petviashvili. Equation of an extraordinary soliton(ion acoustic wave packet dispersion in plasma). Sov. J. Plasma Phys., 2:257-258, 1976.

[41] M.E. Schonbek. Existence of solutions for the Boussinesq system of equations. Journal of Differential Equations, 42(3):325-352, 1981.

[42] A. Senthikumar. On the influence of wave reflection on shoaling and breaking solitary waves. Proceedings of the Estonian Academy of Sciences, 65:414-430, 2016.

[43] O. Sørensen, H. Schäffer, and L. Sørensen. Boussinesq-type modelling using an unstructured finite element technique. Coastal Engineering, 50:181-198, 2004.

[44] M. Walkley and M. Berzins. A finite element method for the one-dimensional extended Boussinesq equations. Int. J. Num. Meth. Fluids, 29(2):143-157, 1999.

[45] M. Walkley and M. Berzins. A finite element method for the two-dimensional extended Boussinesq equations. Int. J. Num. Meth. Fluids, 39:865-885, 2002.

[46] G. Wei and J. Kirby. Time-dependent numerical code for extended Boussinesq equations. J. Waterway Port Coastal and Oc Eng, 121:251-261, 1995.

[47] G.B. Whitham. Linear and nonlinear waves, volume 42. John Wiley \& Sons, New York, 2011.

[48] Z. Zhong and K. Wang. Time-accurate stabilized finite-element model for weakly nonlinear and weakly dispersive water waves. Int. J. Num. Meth. Fluids, 57:715-744, 2008. 
Cemse, Kaust, Thuwal, Saudi Arabia, \& Dept. of Math. and Applied Mathematics, Univ. of Crete, Heraklion, Greece, \& iACM, Forth, Heraklion, Greece

Email address: theodoros.katsaounis@kaust.edu.sa

School of Mathematics and Statistics, Victoria University of Wellington, Wellington, New Zealand Email address: dimitrios.mitsotakis@vuw.ac.nz

Laboratoire de Mathématiques Raphä̈l Salem, Université de Rouen Normandie, CNRS UMR 6085, Avenue de L'Université, BP 12, F-76801 Saint-Étienne-Du-Rouvray, France

Email address: georges.sadaka@u-picardie.fr 